Document downloaded from:

http://hdl.handle.net/10251/103794

This paper must be cited as:

Behl, R.; Cordero Barbero, A.; Motsa, SS.; Torregrosa Sánchez, JR. (2017). Stable highorder iterative methods for solving nonlinear models. Applied Mathematics and Computation. 303:70-88. doi:10.1016/j.amc.2017.01.029

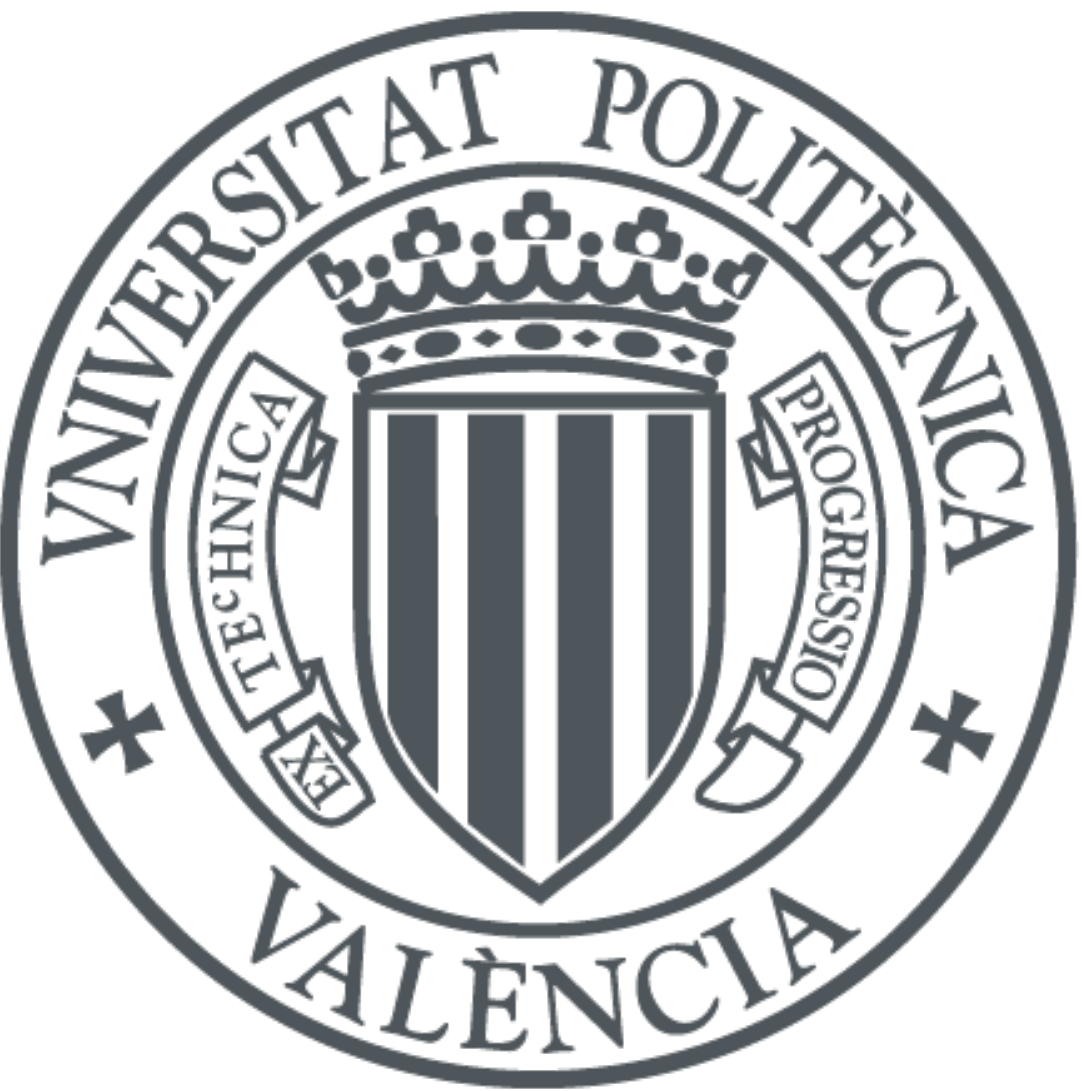

The final publication is available at

http://dx.doi.org/10.1016/j.amc.2017.01.029

Copyright Elsevier

Additional Information 


\title{
Stable high-order iterative methods for solving nonlinear models th
}

\author{
Ramandeep Behl ${ }^{\mathrm{a}}$, Alicia Cordero ${ }^{\mathrm{b}, *}$, Sandile S. Motsa ${ }^{\mathrm{a}}$, Juan R. Torregrosa ${ }^{\mathrm{b}}$ \\ a School of Mathematical Sciences, University of KwaZulu-Natal, Private Bag X01, Scottsville 3209, Pietermaritzburg, South Africa \\ ${ }^{b}$ Instituto Universitario de Matemática Multidisciplinar, Universitat Politècnica de València, València, Spain
}

\begin{abstract}
There are several problems of pure and applied science which can be studied in the unified framework of the scalar and vectorial nonlinear equations. In this paper, we propose a sixth-order family of Jarratt type methods for solving nonlinear equations. Further, we extend this family to the multidimensional case preserving the order of convergence. Their theoretical and computational properties are fully investigated along with two main theorems describing the order of convergence. We use complex dynamics techniques in order to select, among the elements of this class of iterative methods, those more stable. This process is made by analyzing the conjugacy class, calculating the fixed and critical points and getting conclusions from parameter and dynamical planes. For the implementation of the proposed schemes for system of nonlinear equations, we consider some applied science problems namely, Van der Pol problem, kinematic syntheses, etc. Further, we compare them with existing sixth-order methods to check the validity of the theoretical results. From the numerical experiments, we find that our proposed schemes perform better than the existing ones. Further, we also consider a variety of nonlinear equations to check the performance of the proposed methods for scalar equations.
\end{abstract}

Keywords: Nonlinear systems; iterative methods; convergence; basin of attraction; parameter plane; stability.

\section{Introduction}

The design of fixed point iterative methods for obtaining the solutions of equations and systems of nonlinear equations is an important and challenging task in the field of numerical analysis. In 1990, Moré [25] proposed a collection of nonlinear model problems and most of them are phrased in the terms of $F(x)=0$. On the other hand, Grosan and Abraham [19], also discussed the applicability of the system of nonlinear equations in neurophysiology, kinematics syntheses problem, chemical equilibrium problem, combustion problem and economics modeling problem. In addition, the reactor and steering problems are solved in [6, 34] by phrasing these problems in the form of $F(x)=0$. Moreover, Lin et al. [22] also discussed the applicability of the system of nonlinear equations in transport theory.

Analytical methods to obtain the exact solution of such problems are almost non existent. Therefore, many researchers around the world are designing new iterative methods for solving this kind of problems. Generally, these iterative methods provide an approximated solution corrected up to a specified degree of accuracy which is further depend upon the methods and programming software namely, Fortran, Maple, Matlab, Mathematica, etc. While, using these iterative schemes, researchers have to face several problems and some of them are related to slow convergence, non-convergence, oscillation problem close to the initial guess, divergence, failure etc. (for the detail explanation of these problems please see [27, 33]).

In the past and recent years, researchers proposed a plethora of one point and multi-point methods for obtaining the solutions of nonlinear equations (we can see an overview in [27]) and system of nonlinear equations. Generally, most of them are the extension of the classical Newton method or Newton like method at the expense of some additional functional evaluations of the involved function.

Sometimes researchers extend, in a natural way, methods designed for nonlinear equations to the multidimensional case. For example, recently Cordero et al. [15], implemented fourth-order Jarratt's method [21] for scalar equations to system of nonlinear equations. Further, Abad et al. [1], Cordero et al. [17] and Wang et al. [36], proposed some higher-order extension for systems of nonlinear equations of the previously published work for the scalar equations. We can say that it is one of

\footnotetext{
This research was partially supported by Ministerio de Economía y Competitividad MTM2014-52016-C2-2-P and by Generalitat Valenciana PROMETEO/2016/089.

*Corresponding author

Email addresses: ramanbeh187@yahoo.in (Ramandeep Behl), acordero@mat.upv.es (Alicia Cordero ), sandilemotsa@gmail.com (Sandile S. Motsa ), jrtorreemat.upv.es (Juan R. Torregrosa )
} 
simple way to develop new schemes for solving nonlinear systems. But, it is not always possible at least, in the same form. One of the main reasons behind this is that, in the scalar case, functional evaluation of the involved function and its derivative have the same computational cost. However, this is not true in the multidimensional case.

Therefore, researchers also tried some other approaches and procedures to develop new and higher-order methods for systems of nonlinear equations. Recently, Sharma et al. [31] proposed fourth and six-order iterative methods based on weighted-Newton iteration. Very recently, Artidiello et al. [5] proposed fourth-order methods based on the weight function approach. Different researchers have used quadrature formulae, Adomian polynomial, divided difference approach, ... for constructing iterative schemes to solve nonlinear systems. For the details of the other approaches one refers some standard text books [26, 33].

In this paper, our main aim is to propose a sixth-order family of Jarratt type methods for solving scalar equations. A qualitative analysis is made to select some elements of the family with good stability properties. Then, we extend this family for the multidimensional case, with the same order of convergence. The efficiency of the proposed methods is tested on several numerical examples and it is found that our proposed methods perform better than many existing ones in the scalar case. Further, we have also checked the applicability of our proposed scheme in the multidimensional case on some applied examples.

Now, we are going to recall some concepts related to the convergence of an iterative method.

Definition 1. Let us consider a sequence $\left\{x_{n}\right\}$ converging to a root $\xi$ of $f(x)=0$, where $f$ is a real function. Let $e_{n}=x_{n}-\xi$ be the error at nth iteration. If constants $p \geq 1, c \neq 0$ exist in such a way that $e_{n+1}=c e_{n}^{p}+O\left(e_{n}^{p+1}\right)$, known as the error equation, then $p$ and $\eta=|c|$ are said to be the order of convergence and the asymptotic error constant, respectively (in the linear case, that is $p=1$, it is necessary that the asymptotic constant satisfies $\eta<1$ ). From this definition the asymptotic error constant is found to be $\eta=|c|=\lim _{n \rightarrow \infty} \frac{\left|e_{n+1}\right|}{\left|e_{n}^{p}\right|}$.

With the help of previous concepts, we can define the asymptotic order of convergence as

$$
p=\lim _{n \rightarrow \infty} \frac{\left|e_{n+1} / \eta\right|}{\left|e_{n}^{p}\right|} .
$$

But, the main drawback of calculating $\eta$ according to the above formula is that it involves the exact root $\xi$ and there are many real situations in which the exact root is not known in advance. To overcome this problem, we can use $\left(x_{n+1}-x_{n}\right)$ instead of $\left(e_{n+1}\right)$ in the above formula to calculate $\eta$. Further, in a similar way for systems of nonlinear equations, we can define the error equation, asymptotic error constant, etc. by using any norm in $\mathbb{R}^{n}$.

The rest of the paper is organized as follows: in Section 2 we propose a new sixth-order family of Jarratt type method for obtaining the solutions of nonlinear equations and also give a proof of its order of convergence. In Section 3 , we use tools of complex dynamics in order to analyze the stability of the members of the presented class. Section 4 is devoted to the generalization of the previous family for solving nonlinear systems preserving its order of convergence. In Section 5, we propose the numerical tests that confirm the theoretical results and allow us to compare the proposed schemes with some well known existing methods of order six. We finish the paper with some conclusions and the references used in it.

\section{Development of the scheme for scalar equations}

In this section, we propose a new sixth-order family of Jarratt-type methods. We consider the three-step family

$$
\begin{aligned}
y_{n} & =x_{n}-a \frac{f\left(x_{n}\right)}{f^{\prime}\left(x_{n}\right)}, \\
z_{n} & =y_{n}-\left[\frac{b}{f^{\prime}\left(x_{n}\right)}+\frac{1}{c f^{\prime}\left(x_{n}\right)+d f^{\prime}\left(y_{n}\right)}\right] f\left(x_{n}\right), \\
x_{n+1} & =z_{n}-\left[\frac{g}{f^{\prime}\left(x_{n}\right)}+\frac{1}{e f^{\prime}\left(x_{n}\right)+h f^{\prime}\left(y_{n}\right)}\right] f\left(z_{n}\right),
\end{aligned}
$$

where $a, b, c, d, e, g$ and $h$ are free disposable parameters. The following result demonstrates that the order of convergence reachs sixth-order from some conditions on the parameters. 
Theorem 1. Let $f: I \subseteq \mathbb{R} \rightarrow \mathbb{R}$ be a sufficiently differentiable function in an interval I containing a simple root $\xi$ of the equation $f(x)=0$. Further, let us assume that an initial guess $x_{0}$ is sufficiently close to $\xi$. Then, the iterative scheme defined by (1) has a sixth-order convergence when

$$
a=\frac{2}{3}, \quad b=-\frac{1}{6}, \quad c=-1, d=3, \quad e=-\frac{2 g+1}{2(g-1)^{2}} \quad \text { and } h=\frac{3}{2(g-1)^{2}},
$$

where $g \neq 1$. It satisfies the error equation

$$
e_{n+1}=\frac{\left(9 c_{2}^{3}-9 c_{3} c_{2}+c_{4}\right)\left(c_{2}^{2}(6 g-2)-c_{3}(g-1)\right)}{9(g-1)} e_{n}^{6}+O\left(e_{n}^{7}\right),
$$

where $e_{n}=x_{n}-\xi$ and $c_{j}=\frac{f^{(j)}(\xi)}{j ! f^{\prime}(\xi)}$ for $j=1,2, \ldots$

Proof. Let us consider $e_{y_{n}}=y_{n}-\xi$ and $e_{z_{n}}=z_{n}-\xi$ be the errors in the $n$th iteration. The Taylor's series expansion of function $f\left(x_{n}\right)$ and it's first order derivative $f^{\prime}\left(x_{n}\right)$ around $x=\xi$ with the assumption $f^{\prime}(\xi) \neq 0$ leads us to:

$$
f\left(x_{n}\right)=f^{\prime}(\xi)\left[\sum_{j=1}^{6} c_{j} e_{n}^{j}+O\left(e_{n}^{7}\right)\right]
$$

and

$$
f^{\prime}\left(x_{n}\right)=f^{\prime}(\xi)\left[\sum_{j=1}^{6} j c_{j} e_{n}^{j-1}+O\left(e_{n}^{6}\right)\right]
$$

respectively.

By using the equations (4) and (5) in the first step of scheme (1) we get

$$
e_{y_{n}}=(1-a) e_{n}+a c_{2} e_{n}^{2}+\sum_{k=3}^{6} G_{k} e_{n}^{k}+O\left(e_{n}^{7}\right)
$$

where $G_{k}=G_{k}\left(c_{2}, c_{3}, \ldots, c_{6}\right)$.

Now, the following expansion of $f^{\prime}\left(y_{n}\right)$ about $\xi$ by using (6), is obtained

$$
f^{\prime}\left(y_{n}\right)=f^{\prime}(\xi)\left[\sum_{j=1}^{5} j c_{j} e_{y_{n}}^{j-1}+O\left(e_{y_{n}}^{5}\right)\right]
$$

By inserting (4) - (7) in the second sub-step of the proposed scheme (1) and further simplifying the equations, we get

$$
e_{z_{n}}=\left(1-a-b-\frac{1}{c+d}\right) e_{n}+\left(a-\frac{2 a d}{(c+d)^{2}}+\frac{b(c+d)+1}{c+d}\right) c_{2} e_{n}^{2}+\sum_{k=4}^{6} H_{k} e_{n}^{k}+O\left(e_{n}^{7}\right)
$$

where $H_{k}=H_{k}\left(a, b, c, d, c_{2}, c_{3}, \ldots, c_{6}\right)$.

Now, we can expand function $f\left(z_{n}\right)$ about $\xi$, obtaining

$$
f\left(z_{n}\right)=f^{\prime}(\xi)\left[\sum_{j=1}^{4} c_{j} e_{z_{n}}^{j}+O\left(e_{z_{n}}^{5}\right)\right]
$$

Using equations (4) - (9), in the last step of the proposed scheme (1) and simplifying laster the equations, we obtain

$$
e_{n+1}=\frac{(e(g-1)+(g-1) h+1)(c(a+b-1)+d(a+b-1)+1)}{(c+d)(e+h)} e_{n}+\sum_{k=2}^{6} P_{k} e_{n}^{k}+O\left(e_{n}^{7}\right) \text {, }
$$


where $P_{k}=P_{k}\left(a, b, c, d, e, g, h, c_{2}, c_{3}, \ldots, c_{6}\right)$.

If we substitute the following value of the disposable parameter $d$ in equation (10), then we will get at least quadratic convergence,

$$
d=-\frac{c(a+b-1)+1}{a+b-1} .
$$

Let us insert the above value of $d$ into $P_{2}=0$. Then, we will obtain at least cubic convergence when we consider the following value of $h$

$$
h=\frac{-g e+e-1}{g-1} .
$$

Using equations (11) and (12) into $P_{3}=0$, we obtain the following independent relation

$$
e\left(a(g-1)^{2}\right)+a g+a-1=0 .
$$

After simplifying, we get the following value of $e$

$$
e=\frac{1-a-a g}{a(g-1)^{2}}
$$

In this way, we demonstrate that our proposed scheme reached at least fourth-order of convergence. Further, by substituting the equations (11), (12) and (13) in $P_{4}=0$, we get the following independent relation

$$
2 a^{3} c+a^{2}(4(b-1) c+2)+2 a(b-1)((b-1) c+1)+1=0,
$$

which further yields

$$
c=-\frac{2 a^{2}+2 a(b-1)+1}{2 a(a+b-1)^{2}} .
$$

Now, inserting the equations (11), (12), (13) and (14) in $P_{5}=0$, we obtain two independent relations, which are given by

$$
\left\{\begin{array}{l}
\frac{3 a^{2}(5 g-3)+a(3 b(5 g-3)-24 g+14)+b(8-12 g)+9 g-5}{(g-1)(a+b-1)}=0, \\
\frac{2(3 g-1)(2 a+2 b-1)}{(g-1)(a+b-1)}=0,
\end{array}\right.
$$

By solving (15) for $a$ and $b$, we get

$$
b=-\frac{1}{6}, a=\frac{2}{3} .
$$

By using (11), (12), (13) (14) and (15) into (16), we will get the following error equation

$$
e_{n+1}=\frac{\left(9 c_{2}^{3}-9 c_{3} c_{2}+c_{4}\right)\left(c_{2}^{2}(6 g-2)-c_{3}(g-1)\right)}{9(g-1)} e_{n}^{6}+O\left(e_{n}^{7}\right),
$$

where $g \neq 1$ is a free disposable parameter. The error equation reveals that the proposed scheme (1) reaches the sixth-order convergence. This completes the proof.

\subsection{Special cases}

In this section, we consider some particular cases of the proposed class (1) depending upon the free disposable parameter namely, $g$ which are given as follows:

(i) For $g=\frac{1}{3}$ in the family (1), we get the following new sixth-order scheme of Jarratt-type

$$
\begin{aligned}
y_{n} & =x_{n}-\frac{2}{3} \frac{f\left(x_{n}\right)}{f^{\prime}\left(x_{n}\right)}, \\
z_{n} & =y_{n}-\left[\frac{1}{3 f^{\prime}\left(y_{n}\right)-f^{\prime}\left(x_{n}\right)}-\frac{1}{6 f^{\prime}\left(x_{n}\right)}\right] f\left(x_{n}\right), \\
x_{n+1} & =z_{n}-\left[\frac{1}{3 f^{\prime}\left(x_{n}\right)}-\frac{8}{15 f^{\prime}\left(x_{n}\right)-27 f^{\prime}\left(y_{n}\right)}\right] f\left(z_{n}\right) .
\end{aligned}
$$

It satisfies the following error equation

$$
e_{n+1}=\frac{1}{9} c_{3}\left(-9 c_{2}^{3}+9 c_{3} c_{2}-c_{4}\right) e_{n}^{6}+O\left(e_{n}^{7}\right) .
$$


(ii) For $g=-\frac{1}{2}$ in (1), we obtain

$$
x_{n+1}=z_{n}-\left[\frac{3}{2 f^{\prime}\left(y_{n}\right)}-\frac{1}{2 f^{\prime}\left(x_{n}\right)}\right] f\left(z_{n}\right),
$$

where $y_{n}$ and $z_{n}$ are the same as in (18). This is another new Jarratt-type method of order six, whose error equation is

$$
e_{n+1}=\frac{1}{27}\left(10 c_{2}^{2}-3 c_{3}\right)\left(9 c_{2}^{3}-9 c_{3} c_{2}+c_{4}\right) e_{n}^{6}+O\left(e_{n}^{7}\right)
$$

(iii) For $g=\frac{34}{100}$ in class (1), we further get another sixth-order Jarratt-type method

$$
x_{n+1}=z_{n}-\left[\frac{34}{100 f^{\prime}\left(x_{n}\right)}-\frac{363}{700 f^{\prime}\left(x_{n}\right)-1250 f^{\prime}\left(y_{n}\right)}\right] f\left(z_{n}\right),
$$

where $y_{n}$ and $z_{n}$ are the same as in (18), satisfying the following error equation

$$
e_{n+1}=-\frac{1}{297}\left(\left(2 c_{2}^{2}+33 c_{3}\right)\left(9 c_{2}^{3}-9 c_{3} c_{2}+c_{4}\right)\right) e_{n}^{6}+O\left(e_{n}^{7}\right) \text {. }
$$

(iv) For $g=0$ we get a more simple third step than in the previous cases, as the inverse to be calculated in this step is the same as one of the second step. This is a key fact for solving nonlinear systems, as the computational cost involved decrease significatively. Specifically,

$$
x_{n+1}=z_{n}-\left[\frac{2}{3 f^{\prime}\left(y_{n}\right)-f^{\prime}\left(x_{n}\right)}\right] f\left(z_{n}\right),
$$

where $y_{n}$ and $z_{n}$ are the same as in (18). Moreover, its error equation is

$$
e_{n+1}=\frac{1}{9}\left(\left(9 c_{2}^{3}-9 c_{3} c_{2}+c_{4}\right)\left(2 c_{2}^{2}-c_{3}\right)\right) e_{n}^{6}+O\left(e_{n}^{7}\right)
$$

We use these particular elements of family (1) in the numerical section and we see in the next section that these values of parameter correspond to stable members of the family.

\section{Dynamical behavior of the family}

The analysis of the complex dynamics of iterative methods for solving nonlinear equations is an expanding area of research that gives us relevant information about the behavior of specific iterative schemes and also help us to select those whose performance on quadratic polynomials show to be more stable than the rest of elements of a family. For the sake of the simplicity of these functions, if the iterative procedure gives unstable results on them, they can be discarded and the most stable ones use to behave in the same manner when applied on other kind of functions (see, among others, [2, 3, 4, 7, 12, 14, 23]).

In order to analyze the dynamical behavior of the rational function obtained when an iterative method is applied on a polynomial $p(z)$, it is necessary to recall some basic dynamical concepts. For a deeper insight, see [8].

Let $R: \hat{\mathbb{C}} \rightarrow \hat{\mathbb{C}}$ be a rational function, where $\hat{\mathbb{C}}$ is the Riemann sphere. The orbit of a point $z_{0} \in \hat{\mathbb{C}}$ is defined as

$$
\left\{z_{0}, R\left(z_{0}\right), \ldots, R^{2}\left(z_{0}\right), \ldots, R^{n}\left(z_{0}\right), \ldots\right\} .
$$

The dynamical behavior of the orbit of a point can be classified depending on its asymptotic behavior on the initial estimation used. In this terms, a point $z^{*} \in \hat{\mathbb{C}}$ is a fixed point of $R$ if $R\left(z^{*}\right)=z^{*}$. If a fixed point is not a root of $p(z)$, it is called strange fixed point. Moreover, a periodic point $z$ of period $p>1$ is a point satisfying $R^{p}(z)=z$ and $R^{k}(z) \neq z$, $k<p$. A pre-periodic point $z$ is not a periodic point but there exists $k>0$ such that $R^{k}(z)$ is a periodic point.

So, the behavior of a fixed point $z^{*}$ depends of the value of the stability function $\left|R^{\prime}\left(z^{*}\right)\right|$ on it, that is called multiplier of the fixed point. Then, a fixed point $z^{*}$ is called

- attracting if its multiplier is lower than one, $\left|R^{\prime}\left(z^{*}\right)\right|<1$,

- superattracting when it is null, $\left|R^{\prime}\left(z^{*}\right)\right|=0$,

- repelling if $\left|R^{\prime}\left(z^{*}\right)\right|>1$ or 
- neutral or parabolic when $\left|R^{\prime}\left(z^{*}\right)\right|=1$.

From the point of view of the convergence, let us remark that the roots of $p(z)$ will be always superattracting fixed points if the order of convergence of the iterative method is, at least, two.

Also, the stability of a periodic orbit is defined by the magnitude of its multiplier, $\left|R^{\prime}\left(z_{1}\right) \cdots R^{\prime}\left(z_{p}\right)\right|$, being $\left\{z_{1}, \ldots, z_{p}\right\}$ those points defining the periodic orbit of period $p$.

On the other hand, a point $z$ is defined as critical if it satisfies $R^{\prime}(z)=0$. Moreover, if it is not a (superattracting) root of $p(z)$, it is called free critical point.

Let $z^{*}$ be an attracting fixed point of rational function $R$. The basin of attraction of $z^{*}, \mathcal{A}\left(z^{*}\right)$, is defined as the set of pre-images of any order such that

$$
\mathcal{A}\left(z^{*}\right)=\left\{z_{0} \in \hat{\mathbb{C}}: R^{n}\left(z_{0}\right) \rightarrow z^{*}, n \rightarrow \infty\right\} .
$$

On the other hand, Fatou set is composed by those points whose orbits tend to an attractor (fixed point, periodic orbit or infinity). The complementary set, the Julia set $\mathcal{J}(R)$, is the closure of the set consisting of its repelling fixed points or orbits and their preimages, and establishes the borders between the basins of attraction.

Mayer and Schleicher defined in [24] the immediate basin of attraction of an attracting fixed point $z^{*}$ (considered as a periodic point of period 1), as the connected component of the basin containing $z^{*}$. This concept is directly related with the existence of critical points stated early by Fatou and Julia.

Theorem 2. Let $R$ be a rational function. The immediate basin of attraction of any attracting periodic point holds, at least, a critical point.

\subsection{Conjugacy classes}

It is possible to analyze the behavior of an iterative method acting on any quadratic polynomial.

Let $f$ and $g$ be defined as $f, g: \hat{\mathbb{C}} \rightarrow \hat{\mathbb{C}}$. An analytic conjugation between $f$ and $g$ is a diffeomorphism $h: \hat{\mathbb{C}} \rightarrow \hat{\mathbb{C}}$ such that $h \circ f=g \circ h$.

It is easy to prove the following result where the rational functions resulting from the iteration of any method of the family (1) on affine-conjugated analytic functions are also conjugated and so, their dynamics are equivalent.

Theorem 3. (Scaling Theorem) Let $g(z)$ be an analytic function, and let $A(z)=\alpha z+\beta$, with $\alpha \neq 0$, be an affine map. Let $h(z)=\lambda(g \circ A)(z)$, with $\lambda \neq 0$. Let $O_{g}(z)$ be the fixed point operator of family (1) on $g$. Then, $\left(A \circ O_{h} \circ A^{-1}\right)(z)=O_{g}(z)$, i.e. $O_{g}$ and $O_{h}$ are affine conjugated by $A$.

In particular, the rational function associated to the sixth-order family (1) on a generic quadratic polynomial with simple roots $p(z)=(z-a)(z-b)$ is

$$
O_{p}(z)=z-\frac{2}{3} \frac{p(z)}{p^{\prime}(z)}-\left(-\frac{1}{6 p^{\prime}(z)}+\frac{1}{3 p^{\prime}\left(z-2 / 3 \frac{p(z)}{p^{\prime}(z)}\right)-p^{\prime}(z)}\right) p(z) .
$$

Moreover, by applying Möbius map

$$
h(z)=\frac{z-a}{z-b}
$$

satisfying the following properties: $h(\infty)=1, h(a)=0, h(b)=\infty$, the points 0 and $\infty$ play the role of the roots of the polynomial $a$ and $b$ in $O_{p}$ and also the rational operator associated to the class of iterative schemes is free of parameters $a$ and $b$,

$$
M(z)=\left(h \circ O_{p} \circ h^{-1}\right)(z)=z^{6} \frac{-(1+z)^{2}\left(2+z^{2}\right)+g\left(6+8 z+7 z^{2}+4 z^{3}+z^{4}\right)}{-(1+z)^{2}\left(1+2 z^{2}\right)+g\left(1+4 z+7 z^{2}+8 z^{3}+6 z^{4}\right)}
$$

depending exclusively on the free parameter of the family $g \in \hat{\mathbb{C}}$. By this reason, we will denote in the following this rational function by $M(z, g)$.

In order to start the dynamical analysis of the family, we calculate the fixed points of $M(z, g)$, establish their stability (which depend on $g$ ) and then we proceed with the calculation of the critical points, which will allow us to define the different regions of stability. 
Proposition 1. The fixed points of operator $M(z, g)$ are $z=0, z=\infty, z=1$ and the roots of polynomial $q(z)=-1+g+$ $(-3+5 g) z+(-6+12 g) z^{2}+(-10+20 g) z^{3}+(-12+26 g) z^{4}+(-10+20 g) z^{5}+(-6+12 g) z^{6}+(-3+5 g) z^{7}+(-1+g) z^{8}$, that are denoted by $r_{i}(g), i=1,2, \ldots, 8$. The number of strange fixed points is lower if $g=1$; in this case, the rational function is

$$
M(z, 1)=z^{5} \frac{2+2 z+2 z^{2}+z^{3}}{1+2 z+2 z^{2}+2 z^{3}},
$$

where it can be observed that the order of convergence has been reduced to 5 and the strange fixed points are $z=1$ and the roots of polynomial $\left(1+z(1+z)^{2}\right)\left(1+z\left(2+z+z^{2}\right)\right)$.

Now, we analyze the stability of these strange fixed points in terms of parameter $g$.

Theorem 4. The character of the strange fixed point $z=1$ is:

i) Attracting, if $\left|g-\frac{1970}{3927}\right| \leq \frac{32}{3927}$, being superattracting if $g=\frac{1}{2}$.

ii) Parabolic, when $\left|g-\frac{1970}{3927}\right|=\frac{32}{3927}$.

iii) Repulsive, if $\left|g-\frac{1970}{3927}\right| \geq \frac{32}{3927}$

Proof. It is easy to prove that

$$
M^{\prime}(1, g)=\frac{32-64 g}{6-13 g}
$$

Then, the condition to be checked in order to characterize an attracting or parabolic point is

$$
\left|\frac{32-64 g}{6-13 g}\right| \leq 1, \quad \text { equivalent to } \quad 32|1-2 g| \leq|6-13 g|
$$

By considering $g=a+i b$ an arbitrary complex number,

$$
1024\left(1-4 a+4 a^{2}+4 b^{2}\right) \leq 36-156 a+169 a^{2}+169 b^{2} .
$$

By simplifying

$$
3927 a^{2}+3927 b^{2}-3940 a+988 \leq 0
$$

that is,

$$
\left(a-\frac{1970}{3927}\right)^{2}+b^{2} \leq\left(\frac{32}{3927}\right)^{2}
$$

Therefore,

$$
\left|M^{\prime}(1, g)\right| \leq 1 \text { if and only if }\left|g-\frac{1970}{3927}\right| \leq \frac{32}{3927}
$$

Finally, if $g$ satisfies $\left|g-\frac{1970}{3927}\right|>\frac{32}{3927}$, then $\left|M^{\prime}(1, g)\right|>1$ and $z=1$ is a repulsive point.

The stability function of $z=1$ set in Theorem 4 can be observed graphically in Figure 1 as a blue three-dimensional region, showing the complex values of $g$ where $\left|M^{\prime}(1, g)\right|<1$.

In fact, the analysis of the stability regions of the rest of strange fixed points yield to different areas of the complex plane where some of them are attracting: these complex values of parameter $g$ are those inside the basis of the cones in the 3-D representation of Figure 2. The values of $g$ corresponding to the vertices of these cones correspond to $\left|M^{\prime}\left(r_{i}(g), g\right)\right|=0$, for some $i \in\{1,2, \ldots, 8\}$, that is, where these specific strange fixed points are superattracting. In Table 1, the approximate values of $g$ where the strange fixed points $r_{i}(g)$ are superattracting are shown. 


\subsection{Parameter and dynamical planes}

As we know, the dynamical behavior of fixed point operator $M(z, g)$ depends on the values of the parameter $g$. Moreover, a key fact to detect any qualitative change is the existence of critical points different from 0 and $\infty$ (the image by the conjugacy map $h$ of the roots of the polynomial), as each invariant Fatou component must hold at least one critical point. Then, these points must be calculated (by solving the equation $M^{\prime}(z, g)=0$ ) and used as initial estimations of the iteration function in order to detect all the possible basins of attraction and construct a global map of the behavior of $M(z, g)$.

Lemma 1. Points $z= \pm i$ and $z=-1$ are free critical points for any value of $g$. All of them are pre-periodic points, as they are pre-images of $z=1$ by $M(z, g)$. Moreover,

a) If $g=1$, there are two more free conjugated critical points, $\frac{1}{5} i(2 i \pm \sqrt{21})$.

b) If $g=1 / 3$, then there are also two free conjugated critical points, $\frac{1}{7}(2 \pm 3 \sqrt{5} i)$.

c) In any other case, there exists other four free critical points
(i) $c r_{1}(g)=\frac{1}{6}\left(\frac{-13 g^{2}+11 g-3}{3 g^{2}-4 g+1}-R_{1}(g)-\sqrt{2} \frac{\sqrt{-65 g^{4}+278 g^{3}-269 g^{2}+90 g-9+R_{1}(g)\left(39 g^{4}-85 g^{3}+66 g^{2}-23 g+3\right)}}{3 g^{2}-4 g+1}\right)$
(ii) $c r_{2}(g)=\frac{1}{6}\left(\frac{-13 g^{2}+11 g-3}{3 g^{2}-4 g+1}-R_{1}(g)+\sqrt{2} \frac{\sqrt{-65 g^{4}+278 g^{3}-269 g^{2}+90 g-9+R_{1}(g)\left(39 g^{4}-85 g^{3}+66 g^{2}-23 g+3\right)}}{3 g^{2}-4 g+1}\right)$
(iii) ${ } r_{3}(g)=\frac{1}{6}\left(\frac{-13 g^{2}+11 g-3}{3 g^{2}-4 g+1}+R_{1}(g)-\sqrt{2} \frac{\sqrt{-65 g^{4}+278 g^{3}-269 g^{2}+90 g-9-R_{1}(g)\left(39 g^{4}-85 g^{3}+66 g^{2}-23 g+3\right)}}{3 g^{2}-4 g+1}\right)$
(iv) $\operatorname{cr}_{4}(g)=\frac{1}{6}\left(\frac{-13 g^{2}+11 g-3}{3 g^{2}-4 g+1}+R_{1}(g)+\sqrt{2} \frac{\sqrt{-65 g^{4}+278 g^{3}-269 g^{2}+90 g-9-R_{1}(g)\left(39 g^{4}-85 g^{3}+66 g^{2}-23 g+3\right)}}{3 g^{2}-4 g+1}\right)$
where $R_{1}(g)=\frac{\sqrt{25 g^{4}-22 g^{3}+55 g^{2}-42 g+9}}{3 g^{2}-4 g+1}$, satisfying $c r_{1}(g)=\frac{1}{c r_{2}(g)}$ and $c r_{3}(g)=\frac{1}{c r_{4}(g)}$.

From the previous result, let us remark that

- If $g=\frac{6}{13}$, then

$$
M(z, g)=-z^{6} \frac{7 z^{3}+9 z^{2}+6 z+10}{10 z^{3}+6 z^{2}+9 z+7}
$$

and $z=1$ is not a fixed point and critical points $z= \pm i$ and $z=-1$ are not pre-periodic. In this case, there exist two more free conjugated critical points, $\frac{1}{35} i(4 \pm \sqrt{1209} i)$. Moreover, all the strange fixed points (roots of the polynomial $\left.7 z^{8}+9 z^{7}+6 z^{6}+10 z^{5}+10 z^{3}+6 z^{2}+9 z+7\right)$ are repulsive.

- When $g=\frac{1}{2}, z= \pm 1$ are the only free critical points, and the associated operator is $M(z, 1 / 2)=-z^{6} \frac{-2+z^{2}}{-1+2 z^{2}}$.

- If $g=0$, then the associated operator is $M(z, 0)=z^{6} \frac{2+z^{2}}{1+2 z^{2}}$ and the only free critical points are $z= \pm i$, pre-images of $z=1$ that is a repulsive fixed point.

- For $g=1$, then $M(z, 1)=z^{5} \frac{2+2 z+2 z^{2}+z^{3}}{1+2 z+2 z^{2}+2 z^{3}}$ and the corresponding method has order of convergence five.

- However, if $g=\frac{1}{3}$, the fixed point rational function is $M(z, 1 / 3)=z^{7} \frac{2+z+z^{2}+z^{3}}{1+z+z^{2}+z^{3}}$ and the corresponding method has order of convergence seven, for quadratic polynomials. 
- For the values of parameter

$$
\begin{aligned}
& g=\frac{1}{150}\left(33-\sqrt{-7161+\frac{221475}{R_{2}(g)}+75 R_{2}(g)}-\sqrt{3} \sqrt{-4774-\frac{73825}{R_{2}(g)}-25 R_{2}(g)-\frac{74736 \sqrt{3}}{\sqrt{-2387+\frac{73825}{R_{2}(g)}+25 R_{2}(g)}}},,\right. \\
& g=\frac{1}{150}\left(33-\sqrt{-7161+\frac{221475}{R_{2}(g)}+75 R_{2}(g)}+\sqrt{3} \sqrt{\left.-4774-\frac{73825}{R_{2}(g)}-25 R_{2}(g)-\frac{74736 \sqrt{3}}{\sqrt{-2387+\frac{73825}{R_{2}(g)}+25 R_{2}(g)}}\right)},\right. \\
& g=\frac{1}{150}\left(33+\sqrt{-7161+\frac{221475}{R_{2}(g)}+75 R_{2}(g)}-\sqrt{3} \sqrt{-4774-\frac{73825}{R_{2}(g)}-25 R_{2}(g)-\frac{74736 \sqrt{3}}{\sqrt{-2387+\frac{73825}{R_{2}(g)}+25 R_{2}(g)}}}\right), \\
& g=\frac{1}{150}\left(33+\sqrt{-7161+\frac{221475}{R_{2}(g)}+75 R_{2}(g)}+\sqrt{3} \sqrt{-4774-\frac{73825}{R_{2}(g)}-25 R_{2}(g)-\frac{74736 \sqrt{3}}{\sqrt{-2387+\frac{73825}{R_{2}(g)}+25 R_{2}(g)}}}\right),
\end{aligned}
$$

where $R_{2}(g)=(146341+1296 i \sqrt{2581})^{1 / 3}, c r_{1}(g)=c r_{2}(g)=c r_{3}(g)=c r_{4}(g)$. So, there is only one free critical point that is not a pre-periodic point.

Free critical points of rational function $M(z, g)$ are closely related to parameter space. It is obtained by associating each point of the parameter plane with a complex value of $g$, that is, with an element of family of iterative schemes (1). Every value of $g$ belonging to the same connected component of the parameter space gives rise to subsets of schemes of family (1) with similar dynamical behavior. So, it is interesting to find regions of the parameter plane as much stable as possible, because these values of $g$ will give us the best members of the family in terms of numerical stability.

In this terms, we must recall that, in general, there exist only two free independent critical points: three free critical points are pre-periodic, so the only information that they give is the stability of the fixed point whose they are pre-images. The other four ones, depending on parameter $g$ are conjugated two-by-two. Therefore, we use $c r_{1}(g)$ and $c r_{3}(g)$ to calculate both parameter planes. Firstly, a mesh of $2000 \times 2000$ points in the complex plane is defined, corresponding to different values of $g$. When we consider $c r_{1}(g)$ as a starting point of the iterative scheme of the family associated to each complex value of $g$, we paint this point in different colors depending of where they converge to (after a maximum of 80 iterations): it is painted in red if the method converges to any of the roots (zero and infinity) and they are white in other cases. Then, parameter plane $P_{1}$ is obtained; it is showed in Figure 3a. Let us remark that red color is brighter as lower is the number of iterations needed to converge. By using a similar procedure but using $\mathrm{cr}_{3}(g)$ as starting point, parameter plane $P_{2}$ is obtained (see Figure $3 \mathrm{c}$ ).

Let us observe that white regions are extremely small in both cases, and some of them correspond to the stability regions of strange fixed points showed in Figure 2, as can be observed in the details of both pictures. In Figure 3b, the two bigger disks correspond, respectively, to the stability regions of strange fixed points $z=1$ (the biggest) and $r_{3}(g)$ and $r_{4}(g)$; in the later case, both fixed points are attracting simultaneously for the same values of parameter $g$. Regarding Figure $3 \mathrm{~d}$, the biggest white area correspond to a deformation of the stability region of $z=1$, meanwhile the small Mandelbrot set at the right of the image corresponds to the stability region of strange fixed points $r_{7}(g)$ and $r_{8}(g)$. The rest of white regions correspond to other kind of unstable behavior, as the stability regions of periodic orbits of different periods.

In order to detect and classify these sets of values of $g$ with unstable behavior (and, therefore, the widest regions of stable performance), we use the dynamical planes. In them, we represent the convergence basins of specific members of the parametric family by using the software described in [13]. We fix a value of parameter $g$, that is, a method of family; then, we draw a mesh with eight hundred points per axis; each point of the mesh is a different complex initial estimation which we introduce in the procedure fixed by $g$. When the method converges to $z=0$ in less than eighty iterations, this point is drawn in orange; if it converges to $z=\infty$, it is painted in blue color; convergence to attracting strange fixed points are painted in green, red,... The color is more intense when the number of iterations is lower. Otherwise, if the method arrives at the maximum of iterations, the point is drawn in black. Usually this behavior corresponds to attracting periodic orbits.

Some dynamical planes corresponding to values of $g$ in white regions of parameter plane $P_{1}$ (Figure $3 \mathrm{~b}$ ) and $P_{2}$ (Figure 3c) are presented in Figure 4.

In particular, Figures $4 \mathrm{a}$ and $4 \mathrm{~b}$ show two 2-periodic orbits (their orbits are presented with yellow lines) corresponding to the a bulb in the left-hand side of the white structure in Figure 4c. In the following white disk (moving to bigger values of $g$ ) we take $g=0.49$, where dynamical plane in Figure $4 \mathrm{c}$ show a unique attracting 2-periodic orbit. The next bulb to the righthand side corresponds to the stability region of $z=1$, and it can be observed in the corresponding dynamical plane (Figure $4 \mathrm{~d}$ ), that this point has its own basin of attraction. The following white disk at the right sets the stability region of strange 
fixed points $r_{3}(g)$ and $r_{4}(g)$, whose respective basins of attractions are shown (red and green colors) in Figure 4e. Figure 4f corresponds to $g=0.5217$, where the two previous attracting fixed points have bifurcated in two attracting 2-periodic orbits, whose basins of attraction lay at the black areas at both sides of $z=1$. Last row of figures correspond to two 3-periodic orbits (Figures $4 \mathrm{~g}$ and $4 \mathrm{~h}$ ), meanwhile in Figure $4 \mathrm{i}$ one of the two existing 6-periodic orbits is shown, for a complex value of $g$. The relevance of finding 3-periodic orbits yields in Sarkovski's theorem, that stablishes the existence of orbits of any periodicity if there exists a 3-periodic orbit (see [8]).

However, the most common behavior is stability, as it is deduced from parameter planes, where the red stable regions domain. In Figure 5, some of these red values have been selected and their respective dynamical planes have been obtained. There, the most stable values that have been detected in the analysis of previous section appear, joint with some others. It is relevant that in many of them a clear behavior similar to Newton's method is showed, but with 6th-order (or even 7th-order on quadratic polynomials) of convergence. Some of these stable elements of the family will be used in the numerical section to effectively check the performance of the family on non-polynomial functions.

\section{Development of the family for nonlinear systems}

We can extend family (1) for solving a nonlinear system $F(x)=0$, where $F: D \subseteq \mathbb{R}^{n} \rightarrow \mathbb{R}^{n}$ is the function that describes the system, in a following way:

$$
\begin{aligned}
y^{(k)} & =x^{(k)}-a F^{\prime}\left(x^{(k)}\right)^{-1} F\left(x^{(k)}\right), \\
z^{(k)} & =y^{(k)}-\left[b F^{\prime}\left(x^{(k)}\right)^{-1}+\left(c F^{\prime}\left(x^{(k)}\right)+d F^{\prime}\left(y^{(k)}\right)\right)^{-1}\right] F\left(x^{(k)}\right), \\
x^{(k+1)} & =z^{(k)}-\left[g F^{\prime}\left(x^{(k)}\right)^{-1}+\left(e F^{\prime}\left(x^{(k)}\right)+h F^{\prime}\left(y^{(k)}\right)\right)^{-1}\right] F\left(z^{(k)}\right),
\end{aligned}
$$

where $a, b, c, d, e, g$ and $h$ are free disposable parameters. With the values of the parameters obtained in Theorem 1 we design a parametric family of sixth-order iterative methods for solving nonlinear systems as shows the following result. In order to clarify its proof, we remember in the following some notions and results introduced in [15].

Let $F: D \subseteq \mathbb{R}^{n} \longrightarrow \mathbb{R}^{n}$ be sufficiently differentiable in $D$. The $q$ th derivative of $F$ at $u \in \mathbb{R}^{n}, q \geq 1$, is the $q$-linear function $F^{(q)}(u): \mathbb{R}^{n} \times \cdots \times \mathbb{R}^{n} \longrightarrow \mathbb{R}^{n}$ such that $F^{(q)}(u)\left(v_{1}, \ldots, v_{q}\right) \in \mathbb{R}^{n}$. It is easy to observe that

1. $F^{(q)}(u)\left(v_{1}, \ldots, v_{q-1}, \cdot\right) \in \mathcal{L}\left(\mathbb{R}^{n}\right)$

2. $F^{(q)}(u)\left(v_{\sigma(1)}, \ldots, v_{\sigma(q)}\right)=F^{(q)}(u)\left(v_{1}, \ldots, v_{q}\right)$, for all permutation $\sigma$ of $\{1,2, \ldots, q\}$.

From the above properties we can use the following notation:

(a) $F^{(q)}(u)\left(v_{1}, \ldots, v_{q}\right)=F^{(q)}(u) v_{1} \ldots v_{q}$

(b) $F^{(q)}(u) v^{q-1} F^{(p)} v^{p}=F^{(q)}(u) F^{(p)}(u) v^{q+p-1}$

On the other hand, for $\xi+h \in \mathbb{R}^{n}$ lying in a neighborhood of a solution $\xi$ of $F(x)=0$, we can apply Taylor's expansion and assuming that the Jacobian matrix $F^{\prime}(\xi)$ is nonsingular, we have

$$
F(\xi+h)=F^{\prime}(\xi)\left[h+\sum_{q=2}^{p-1} C_{q} h^{q}\right]+O\left(h^{p}\right),
$$

where $C_{q}=(1 / q !)\left[F^{\prime}(\xi)\right]^{-1} F^{(q)}(\xi), q \geq 2$. We observe that $C_{q} h^{q} \in \mathbb{R}^{n}$ since $F^{(q)}(\xi) \in \mathcal{L}\left(\mathbb{R}^{n} \times \cdots \times \mathbb{R}^{n}, \mathbb{R}^{n}\right)$ and $\left[F^{\prime}(\bar{x})\right]^{-1} \in \mathcal{L}\left(\mathbb{R}^{n}\right)$.

In addition, we can express $F^{\prime}$ as

$$
F^{\prime}(\xi+h)=F^{\prime}(\bar{x})\left[I+\sum_{q=2}^{p-2} q C_{q} h^{q-1}\right]+O\left(h^{p-1}\right)
$$

where $I$ is the identity matrix. Therefore, $q C_{q} h^{q-1} \in \mathcal{L}\left(\mathbb{R}^{n}\right)$. From (25), we obtain

$$
\left[F^{\prime}(\xi+h)\right]^{-1}=\left[I+X_{2} h+X_{3} h^{2}+X_{4} h^{4}+\cdots\right]\left[F^{\prime}(\xi)\right]^{-1}+O\left(h^{p}\right),
$$


where

$$
\begin{aligned}
& X_{2}=-2 C_{2}, \\
& X_{3}=4 C_{2}^{2}-3 C_{3}, \\
& X_{4}=-8 C_{2}^{3}+6 C_{2} C_{3}+6 C_{3} C_{2}-4 C_{4},
\end{aligned}
$$

We denote $e_{k}=x^{(k)}-\xi$ the error in the $k$ th iteration in the multidimensional case. The equations

$$
e_{k+1}=M e_{k}^{p}+O\left(e_{k}^{p+1}\right)
$$

where $M$ is a $p$-linear function $M \in \mathcal{L}\left(\mathbb{R}^{n} \times \cdots \times \mathbb{R}^{n}, \mathbb{R}^{n}\right)$, is called the error equation and $p$ is the order of convergence. Observe that $e_{k}^{p}$ is $\left(e_{k}, e_{k}, \cdots, e_{k}\right)$.

Theorem 5. Let $F: D \subseteq \mathbb{R}^{n} \rightarrow \mathbb{R}^{n}$ be a sufficiently differentiable function in an open neighborhood $D$ of its zero $\xi$. Let us suppose that $F^{\prime}(x)$ is continuous and nonsingular in $\xi$ and the initial guess $x^{(0)}$ is close enough to $\xi$. Then, the iterative schemes defined by (23) have order of convergence six when

$$
a=\frac{2}{3}, \quad b=-\frac{1}{6}, \quad c=-1, d=3, \quad e=-\frac{2 g+1}{2(g-1)^{2}} \text { and } h=\frac{3}{2(g-1)^{2}},
$$

where $g \neq 1$.

Proof. Firstly, we prove that the first two steps of (23) have order of convergence 4. Let $e_{k}=x^{(k)}-\xi$ be the error of the $k$ th-iteration. Developing $F\left(x^{(k)}\right)$ and $F^{\prime}\left(x^{(k)}\right)$ in a neighborhood of $\xi$, we write

$$
\begin{aligned}
F\left(x^{(k)}\right) & =F^{\prime}(\xi)\left[e_{k}+C_{2} e_{k}^{2}+C_{3} e_{k}^{3}\right]+O\left(e_{k}^{4}\right), \\
F^{\prime}\left(x^{(k)}\right) & =F^{\prime}(\xi)\left[I+2 C_{2} e_{k}+3 C_{3} e_{k}^{2}\right]+O\left(e_{k}^{3}\right),
\end{aligned}
$$

where $I$ is the identity matrix of size $n \times n$ and $C_{k}=\frac{1}{k !} F^{\prime}(\xi)^{-1} F^{(k)}(\xi), k \geq 2$. Inversion of $F^{\prime}\left(x^{(k)}\right)$ yields

$$
F^{\prime}\left(x^{(k)}\right)^{-1}=\left[I+X_{1} e_{k}+X_{2} e_{k}^{2}\right] F^{\prime}(\xi)^{-1},
$$

where $X_{1}=-2 C_{2}$ and $X_{2}=4 C_{2}^{2}-3 C_{3}$. By replacing these expressions in the first step of (23) and using $a=2 / 3$, we have

$$
y^{(k)}-\xi=\frac{1}{3} e_{k}+\frac{2}{3} C_{2} e_{k}^{2}-\frac{2}{3}\left(2 C_{2}^{2}-2 C_{3}\right) e_{k}^{3}+O\left(e_{k}^{4}\right) .
$$

Therefore,

$$
F^{\prime}\left(y^{(k)}\right)=F^{\prime}(\xi)\left[I+\frac{4}{3} C_{2} e_{k}+\frac{1}{3}\left(4 C_{2}^{2}+C_{3}\right) e_{k}^{2}\right]+O\left(e_{k}^{3}\right)
$$

and

$$
-F^{\prime}\left(x^{(k)}\right)+3 F^{\prime}\left(y^{(k)}\right)=F^{\prime}(\xi)\left[2 I+\left(4 C_{2}^{2}-2 C_{3}\right) e_{k}^{2}\right]+O\left(e_{k}^{3}\right) .
$$

Inversion of $-F^{\prime}\left(x^{(k)}+3 F^{\prime}\left(y^{(k)}\right)\right.$ yields

$$
\left[-F^{\prime}\left(x^{(k)}\right)+3 F^{\prime}\left(y^{(k)}\right)\right]^{-1}=\left[\frac{1}{2} I+Y_{1} e_{k}+Y_{2} e_{k}^{2}\right] F^{\prime}(\xi)^{-1}+O\left(e_{k}^{3}\right),
$$

where $Y_{1}=0$ and $Y_{2}=\frac{1}{2}\left(C_{3}-2 C_{2}^{2}\right)$.

In order to determine the order of convergence of the second step of (23) we calculate

$$
-\frac{1}{6} F^{\prime}\left(x^{(k)}\right)^{-1}+\left[-F^{\prime}\left(x^{(k)}\right)+3 F^{\prime}\left(y^{(k)}\right)\right]^{-1}=\left[\frac{1}{3} I+\frac{1}{3} C_{2} e_{k}+\left(C_{3}-\frac{5}{3} C_{2}^{2}\right) e_{k}^{2}\right] F^{\prime}(\xi)^{-1}+O\left(e_{k}^{3}\right)
$$

and

$$
\left[-\frac{1}{6} F^{\prime}\left(x^{(k)}\right)^{-1}+\left[-F^{\prime}\left(x^{(k)}\right)+3 F^{\prime}\left(y^{(k)}\right)\right]^{-1}\right] F\left(x^{(k)}\right)=\left[\frac{1}{3} e_{k}+\frac{2}{3} C_{2} e_{k}^{2}+\frac{1}{3}\left(4 C_{3}-4 C_{2}^{2}\right) e_{k}^{3}\right]+O\left(e_{k}^{4}\right) .
$$


Then, $z^{(k)}-\xi=y^{(k)}-\xi-\left[-\frac{1}{6} F^{\prime}\left(x^{(k)}\right)^{-1}+\left[-F^{\prime}\left(x^{(k)}\right)+3 F^{\prime}\left(y^{(k)}\right)\right]^{-1}\right] F\left(x^{(k)}\right)=O\left(e_{k}^{4}\right)$. So, $z^{(k)}-\xi$ can be written as $z^{(k)}-\xi=M e_{k}^{4}+N e_{k}^{5}+O\left(e_{k}^{6}\right)$, where $M$ and $N$ depend on constants $C_{j}$.

Now, we prove that order six is reached in the third step of (23) with the values of the parameters presented in the hypothesis of the theorem. For this, we develop $F\left(z^{(k)}\right)$ in a neighborhood of $\xi$

$$
F\left(z^{(k)}\right)=F^{\prime}(\xi)\left[M e_{k}^{4}+N e_{k}^{5}\right]+O\left(e_{k}^{6}\right)
$$

In addition, simple algebraic calculations yield

$$
H=\left[-\frac{2 g+1}{2(g-1)^{2}} F^{\prime}\left(x^{(k)}\right)+\frac{3}{2(g-1)^{2}} F^{\prime}\left(y^{(k)}\right)\right]^{-1}=2(1-g)^{2}\left[\frac{1}{2(1-g)} I+Z_{1} e_{k}+Z_{2} e_{k}^{2}\right] F^{\prime}(\xi)^{-1}+O\left(e_{k}^{3}\right),
$$

where $Z_{1}=\frac{g}{(1-g)^{2}} C_{2}$ and $Z_{2}=\frac{(3 g+1)(1-g)}{2(1-g)^{3}} C_{3}+\frac{2 g^{2}+g-1}{(1-g)^{3}} C_{2}^{2}$. Then,

$$
H=\left[(1-g) I+2 g C_{2} e_{k}+\left(\frac{(3 g+1)(1-g)}{1-g} C_{3}+\frac{4 g^{2}-2(1-g)}{1-g}\right) e_{k}^{2}\right] F^{\prime}(\xi)^{-1}+O\left(e_{k}^{3}\right)
$$

Finally,

$$
\left[g F^{\prime}\left(x^{(k)}\right)^{-1}+H\right] F\left(z^{(k)}\right)=M e_{k}^{4}+N e_{k}^{5}+M \frac{(1-g) C_{3}+(6 g-2) C_{2}^{2}}{1-g} e_{k}^{6}+O\left(e_{k}^{7}\right)
$$

and

$$
x^{(k+1)}-\xi=z^{(k)}-\xi-\left[g F^{\prime}\left(x^{(k)}\right)^{-1}+H\right] F\left(z^{(k)}\right)=M \frac{(1-g) C_{3}+(6 g-2) C_{2}^{2}}{1-g} e_{k}^{6}+O\left(e_{k}^{7}\right) .
$$

Therefore, (23) is a parametric family of sixth-order iterative methods.

In the numerical section we apply some elements of this family for solving different problems, comparing the results with that obtained by some known schemes.

\section{Numerical experiments}

This section is devoted to verify the validity and effectiveness of our theoretical results which we have proposed earlier. Further, we want to claim that our proposed modified family of Jarratt's method is competitive with the existing sixth-order methods.

We consider four numerical examples; first one is chosen from Soleymani [32]; second one is chosen from Sharma [28]; third one is chosen from Wang and Liu [35] and fourth one is chosen from Chun [18] with same initial guesses which are mentioned in their papers. Further, we also want to see what will happen if we consider different examples with different initial guesses. To do this, we consider fifth example from Behl et al. [10]. The details of chosen examples or test functions are available in Table 2.

During the current numerical experiments with software Mathematica (Version 9), all computations have been done with multiple precision arithmetic with 1000 digits of mantissa, which minimize round-off errors. Let us remark that, in all tables, $a e(b)$ denotes $a \times 10^{(b)}$.

Now, we employ the new sixth-order methods namely, (18) $\left(M_{6}^{1}\right),(19)\left(M_{6}^{2}\right)$ and (21) $\left(M_{6}^{3}\right)$, to check the effectiveness and validity of the theoretical results. We shall compare our methods with a sixth-order Jarratt's method that is recently proposed by Soleymani [32] (method 14) denoted by $(S M)$. In addition, we compare our methods with a family of improved Jarratt multipoint methods presented by Sharma [28], out of which we consider method (28) (for $a_{1}=1$ ), called by $(S H)$. Moreover, we also consider a family of sixth-order methods proposed by Wang and Liu in [35], out of them we choose their best expression (4) for $(b=1, c=1, d=0)$ denoted by (WLM). Finally, we also compare our methods with some improvements of Jarratt's method with sixth-order convergence designed by Chun [18], out of them we choose method (32) (for $a=-1$ ) denoted by $(C M)$. For better comparisons of our proposed methods, we have displayed the number of iteration indexes $(n)$, approximated zeros $\left(x_{n}\right)$, residual error of the corresponding function $\left(\left|f\left(x_{n}\right)\right|\right)$, errors in the iterations $\left|x_{n}-x_{n-1}\right|$, the approximation of the asymptotic error constant $\eta=\frac{\left|x_{n}-x_{n-1}\right|}{\left|x_{n-1}-x_{n-2}\right|^{6}}$ and the estimation of the order of convergence $\frac{\log \left|\left(x_{n+1}-x_{n}\right) / \eta\right|}{\log \left|x_{n}-x_{n-1}\right|}$ corresponding to each test function in Tables $3-7$. The value of $\eta$ used is the last $\frac{\left|x_{n}-x_{n-1}\right|}{\left|x_{n-1}-x_{n-2}\right|^{6}}$ calculated. 
From Table 3, it is clear that our methods $M_{6}^{1}$ and $M_{6}^{3}$ have a very small residual error in the corresponding function $\left(\left|f_{1}\left(x_{n}\right)\right|\right)$ compared with the other test methods. Further, the errors in the consecutive iterations also demonstrate that our methods converge faster than the existing ones.

In Table 4, we also check the effectiveness of our methods when we consider the same nonlinear equation with same initial approximation which is mentioned by Sharma in [28]. Then, we find that our methods $M_{6}^{1}$ and $M_{6}^{3}$ perform better than $S H$ as well as $W L M$ in terms of absolute error and errors in the consecutive iterations. However, in this particular example our methods don't perform better than $S M$ and $C M$.

Regarding Table 5, we claim that our methods namely, $M_{6}^{1}$ and $M_{6}^{2}$ have a very good performance and they have small residual error in the corresponding function compared with other methods.

It is observed in Table 6 from the above table that our methods namely, $M_{6}^{1}$ and $M_{6}^{2}$ perform better as compare to other existing methods namely, $S H, C M$ and $W L M$ when we compare them with the same nonlinear equation and same initial approximation which is described in Chun [18]. However, in this particular example $S M$ performs slightly better than our methods.

It is straight forward to say (see Table 7) that our methods namely, $M_{6}^{1}$ and $M_{6}^{2}$ also perform better than the existing methods when we compare them with a different example. Further, the errors in the consecutive iterations is a very small quantity which demonstrate that our methods converge faster than the existing methods to the required zero.

In the context of systems of nonlinear equations, we also consider a variety of applied examples to check the validity of theoretical results for nonlinear system. Therefore, we employ the new sixth-order methods namely, (for $g=\frac{1}{3}$, in (23)) $\left(\bar{M}_{6}^{1}\right)$, (for $g=\frac{-1}{2}$, in (23)) $\left(\bar{M}_{6}^{2}\right)$ and (for $g=\frac{34}{100}$, in (23)) $\left(\bar{M}_{6}^{3}\right)$, to verify the performance of these methods on Examples 1 to 3. We compare them with a sixth-order family of iterative method that is recently proposed by Hueso et al. [20], out of them we choose their best expression (14-15) for $\left(t_{1}=-\frac{9}{4}\right.$ and $\left.s_{2}=\frac{9}{8}\right)$ denoted by $\left(H M_{6}\right)$. Moreover, we also compare our methods with sixth-order family of iterative methods designed by Cordero et al. [15, 16], out of them we choose method (6) and method $\left(M_{6}\right)$ denoted by $\left(C M_{6}^{1}\right)$ and $\left(C M_{6}^{2}\right)$, respectively. Finally, we compare our methods with an efficient Jarrattlike methods presented by Sharma [29], we consider method (18) called $\left(S M_{6}\right)$. We give the number of iteration indexes $(n)$, residual error of the corresponding function $\left(\left\|F\left(x^{(k)}\right)\right\|\right)$, error in the iterations $\left\|x^{(k)}-x^{(k-1)}\right\|, \frac{\left\|x^{(k)}-x^{(k-1)}\right\|}{\left\|x^{(k-1)}-x^{(k-2)}\right\| 6}, \eta$ and $\frac{\log \left\|\left(x^{(k)}-x^{(k-1)}\right) / \eta\right\|}{\log \left\|x^{(k-1)}-x^{(k-2)}\right\|}$ in the Tables $8-11$. As in the scalar case, the value of $\eta$ used is the last $\frac{\left\|x^{(k)}-x^{(k-1)}\right\|}{\left\|x^{(k-1)}-x^{(k-2)}\right\|^{6}}$ calculated.

Example 1. Let us consider the Van der Pol equation [11], which is defined as follows:

$$
y^{\prime \prime}-\mu\left(y^{2}-1\right) y^{\prime}+y=0, \mu>0,
$$

which governs the flow of current in a vacuum tube, with the boundary conditions $y(0)=0, y(2)=1$. Further, we consider the partition of the given interval [0,2], which is given by

$$
x_{0}=0<x_{1}<x_{2}<x_{3}<\cdots<x_{n}, \text { where } x_{i}=x_{0}+i h, h=\frac{2}{n} .
$$

Moreover, we assume that

$$
y_{0}=y\left(x_{0}\right)=0, y_{1}=y\left(x_{1}\right), \ldots, y_{n-1}=y\left(x_{n-1}\right), y_{n}=y\left(x_{n}\right)=1 \text {. }
$$

If we discretize the above problem (27) by using the second order divided difference for the first and second derivatives, which are given by

$$
y_{k}^{\prime}=\frac{y_{k+1}-y_{k-1}}{2 h}, y_{k}^{\prime \prime}=\frac{y_{k-1}-2 y_{k}+y_{k+1}}{h^{2}}, k=1,2, \ldots, n-1,
$$

then, we obtain a $(n-1) \times(n-1)$ system of nonlinear equations

$$
2 h^{2} x_{k}-h \mu\left(x_{k}^{2}-1\right)\left(x_{k+1}-x_{k-1}\right)+2\left(x_{k-1}+x_{k+1}-2 x_{k}\right)=0, k=1,2, \ldots, n-1 .
$$

Let us consider $\mu=\frac{1}{2}$ and initial approximation $y_{k}^{(0)}=\log \left(\frac{1}{k^{2}}\right), k=1,2, \ldots, n-1$. In this problem, we consider the value of $n=6$ so that we can obtain a $5 \times 5$ system of nonlinear equations. The solution of this problem is

$$
\xi=(0.8243131 \ldots, 1.516531 \ldots, 2.187958 \ldots, 3.123402 \ldots, 7.824242 \ldots)^{T}
$$

and the results obtained in the numerical estimations appears in Table 8. This table not only shows the applicability of our methods on the real world problem but also methods namely, $\bar{M}_{6}^{1}$ and $\bar{M}_{6}^{3}$ perform better than the existing methods in terms residual error and error in consecutive iterations. 
Example 2. Consider the kinematic synthesis problem for steering as described in [6, 34], in the form system of nonlinear equations, which is given by

$$
\begin{aligned}
& {\left[E_{i}\left(x_{2} \sin \left(\psi_{i}\right)-x_{3}\right)-F_{i}\left(x_{2} \sin \left(\phi_{i}\right)-x_{3}\right)\right]^{2}+\left[F_{i}\left(x_{2} \cos \left(\phi_{i}\right)+1\right)-F_{i}\left(x_{2} \cos \left(\psi_{i}\right)-1\right)\right]^{2}} \\
& -\left[x_{1}\left(x_{2} \sin \left(\psi_{i}\right)-x_{3}\right)\left(x_{2} \cos \left(\phi_{i}\right)+1\right)-x_{1}\left(x_{2} \cos \left(\psi_{i}\right)-x_{3}\right)\left(x_{2} \sin \left(\phi_{i}\right)-x_{3}\right)\right]^{2}=0, \text { for } i=1,2,3,
\end{aligned}
$$

where

$$
E_{i}=-x_{3} x_{2}\left(\sin \left(\phi_{i}\right)-\sin \left(\phi_{0}\right)\right)-x_{1}\left(x_{2} \sin \left(\phi_{i}\right)-x_{3}\right)+x_{2}\left(\cos \left(\phi_{i}\right)-\cos \left(\phi_{0}\right)\right), i=1,2,3
$$

and

$$
F_{i}=-x_{3} x_{2} \sin \left(\psi_{i}\right)+\left(-x_{2}\right) \cos \left(\psi_{i}\right)+\left(x_{3}-x_{1}\right) x_{2} \sin \left(\psi_{0}\right)+x_{2} \cos \left(\psi_{0}\right)+x_{1} x_{3}, i=1,2,3 .
$$

We display the values of $\psi_{i}$ and $\phi_{i}$ (in radians) in Table 9. We consider the initial approximation $x^{(0)}=(0.7,0.7,0.7)$. We obtain the following approximated solution

$$
\xi=(0.9051567 \ldots, 0.6977417 \ldots, 0.6508335 \ldots)^{T}
$$

It is clear from Table 10 that our methods namely, $\bar{M}_{6}^{1}$ and $\bar{M}_{6}^{3}$ converge very fast to the required root compared with the existing ones when we consider an applied science problem like kinematic synthesis problem in terms of residual error and the error in the consecutive iterations.

Example 3. Let us consider the following nonlinear system of nonlinear equation [30]

$$
\left\{\begin{array}{l}
x_{j}^{2} x_{j+1}-1=0,1 \leq j \leq 19 \\
x_{20}^{2} x_{1}-1=0
\end{array}\right.
$$

We choose the initial guess $x^{(0)}=(1.25,1.25, \cdots, 1.25)^{T}$ for this problem for obtaining the required solution $\xi=$ $(1,1, \cdots, 1)^{T}$. The obtained results can be observed in Table 11, where we check the effectiveness and validity of our methods on a system of polynomial equations. It is found that our methods namely, $\bar{M}_{6}^{1}$ and $\bar{M}_{6}^{3}$ perform far better than the existing ones.

\section{Conclusions}

We have constructed a parametric family of sixth-order iterative procedures for solving nonlinear equations. We have used the tools of complex dynamics in order to know which are the most stable members of the class and which of them we must avoid under the numerical point of view. We have extended the mentioned class to the multidimensional case, keeping on the order of convergence. Some academic and real-life examples confirm the theoretical results and show the applicability of the proposed methods.

\section{Acknowledgement}

The authors would like to thank the anonymous reviewers for their useful suggestions and comments.

[1] M.F. Abad, A.Cordero, J.R.Torregrosa, A family of seventh-order schemes for solving nonlinear systems, Bull. Math. Soc. Sci. Math. Roum. 57 (105)(2) (2014) 133-145.

[2] S. Amat, S. Busquier, S. Plaza, Review of some iterative root-finding methods from a dynamical point of view, Sci. Ser. A: Math. Scientia 10 (2004) 3-35.

[3] I.K. Argyros, Á.A. Magreñan, A study on the local convergence and the dynamics of Chebyshev-Halley-type methods free from second derivative, Numer. Algor. 71(1) (2016) 1-23.

[4] I.K. Argyros, Á.A. Magreñan, On the convergence of an optimal fourth-order family of methods and its dynamics, Appl. Math. Comput. 252 (2015) 336-346.

[5] S. Artidiello, A. Cordero, J.R.Torregrosa, M.P. Vassileva, Multidimensional generalization of iterative methods for solving nonlinear problems by means of weight-function procedure, Appl. Math. Comput. 268 (2015) 1064-1071. 
[6] F. Awawdeh, On new iterative method for solving systems of nonlinear equations, Numer. Algor. 54 (2010) $395-409$.

[7] D.K.R. Babajee, A. Cordero, J.R. Torregrosa, Study of iterative methods through the Cayley Quadratic Test, Comput. Appl. Math. 291 (2016) 358-369.

[8] P. Blanchard, Complex Analytic Dynamics on the Riemann Sphere, Bull. AMS 11(1) (1984) 85-141.

[9] R. Behl, Development and analysis of some iterative methods for the solution of nonlinear equations, Ph.D. thesis work.

[10] R. Behl, V. Kanwar, K.K. Sharma, Optimal equi-scaled families of Jarratt's method, Int. J. Comput. Math. 90 (2013) 408-422.

[11] R.L. Burden, J.D. Faires, Numerical Analysis, PWS Publishing Company, Boston, 2001.

[12] F. Chicharro, A. Cordero, J.M. Gutiérrez, J.R. Torregrosa, Complex dynamics of derivative-free methods for nonlinear equations, Appl. Math. Comput. 219 (2013) 7023-7035.

[13] F.I. Chicharro, A. Cordero, Juan R. Torregrosa, Drawing Dynamical and Parameters Planes of Iterative Families and Methods, The Scientific World Journal 2013, Article ID 780153, 11 pages.

[14] A. Cordero, J. García-Maimó, J.R. Torregrosa, M.P. Vassileva, P. Vindel, Chaos in King's iterative family, Appl. Math. Lett. 26 (2013) 842-848.

[15] A. Cordero, J.L. Hueso, E. Martínez, J.R. Torregrosa, A modified Newton-Jarratt's composition, Numer. Algor. 55 (2010), 87-99.

[16] A. Cordero, J.L. Hueso, E. Martínez, J.R. Torregrosa, Increasing the convergence order of an iterative method for nonlinear systems, Appl. Math. Lett. 25 (2012) 2369-2374.

[17] A. Cordero, J.G. Maimó, J.R. Torregrosa, M.P. Vassileva, Solving nonlinear problems by Ostrowski-Chun type parametric families, J. Math. Chem. 52(2014) 430-449.

[18] C. Chun, Some improvements of Jarratt's method with sixth-order convergence, Appl. Math. Comput. 190 (2007) 14321437.

[19] C. Grosan, A. Abraham, A new approach for solving nonlinear equations systems, IEEE Trans. Syst. Man Cybernet Part A: Syst. Humans 38 (2008) 698-714.

[20] J.L. Hueso, E. Martínez, C. Teruel, Convergence, efficiency and dynamics of new fourth and sixth order families of iterative methods for nonlinear systems, Comput. Appl. Math. 275 (2015) 412-420.

[21] P. Jarratt, Some fourth order multipoint iterative methods for solving equations. Math. Comput. 20 (1966) $434-437$.

[22] Y. Lin, L. Bao, X. Jia, Convergence analysis of a variant of the Newton method for solving nonlinear equations, Comput. Math. Appl. 59 (2010) 2121-2127.

[23] Á.A. Magreñán, A new tool to study real dynamics: the convergence plane, Appl. Math. Comput. 248 (2014) $215-224$.

[24] S. Mayer, D. Schleicher, Immediate and Virtual Basins of Newton's Method for Entire Functions, Annales de l'institut Fourier 56(2) (2006) 325-336.

[25] J.J. Moré, A collection of nonlinear model problems, in:E.L. Allgower, K. Georg(Eds.), Computational Solution of Nonlinear Systems of Equations, Lectures in Applied Mathematics, vol.26, Amer. Math. Soc. Providence, RI, 1990 723762.

[26] J.M. Ortega, W.C. Rheinboldt, iterative solution of nonlinear equations in several variables, Academic Press, New-York, 1970.

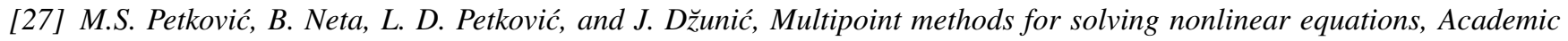
Press, 2012.

[28] J.R. Sharma, A family of improved Jarratt multipoint methods, Int. J. Comput. Math. 84(7) (2007) 1027-1034 . 
[29] J.R. Sharma, H. Arora, Efficient Jarratt-like methods for solving systems of nonlinear equations, Calcolo 51 (2014) 193-210.

[30] J.R. Sharma, H. Arora, A novel derivative free algorithm with seventh order convergence for solving systems of nonlinear equations, Numer. Algor. 67 (2014) 917-933.

[31] J.R. Sharma, R.K. Guna, R. Sharma, An efficient fourth order weighted-Newton method for systems of nonlinear equations, Numer. Algor. 2 (2013) 307-323.

[32] F. Soleymani, Revisit of Jarratt method for solving nonlinear equations, Numer. Algor. 57 (2011) 377-388.

[33] J.F. Traub, Iterative methods for the solution of equations, Prentice-Hall, Englewood Cliffs, 1964.

[34] I.G. Tsoulos, A. Stavrakoudis, On locating all roots of systems of nonlinear equations inside bounded domain using global optimization methods, Nonlinear Anal. Real World Appl. 11 (2010) 2465-2471.

[35] X. Wang, L. Liu, Two new families of sixth-order methods for solving non-linear equations, Appl. Math. Comput. 213 (2009) 73-78.

[36] X. Wang, T. Zhang, A family of Steffensen type methods with seventh-order convergence, Numer. Algor. 62 (2013) 429444. 


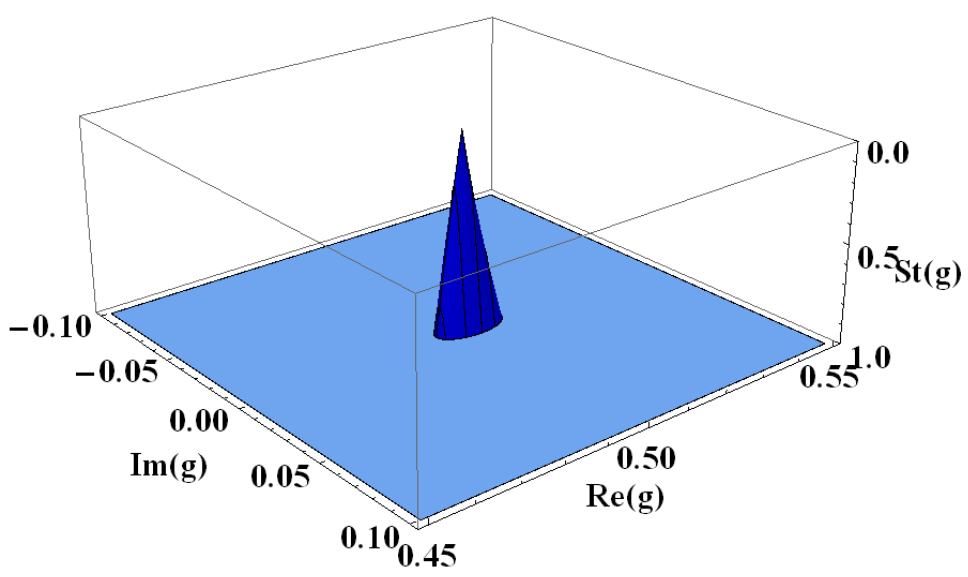

Figure 1: Stability function $\left|M^{\prime}(1, g)\right|$ 


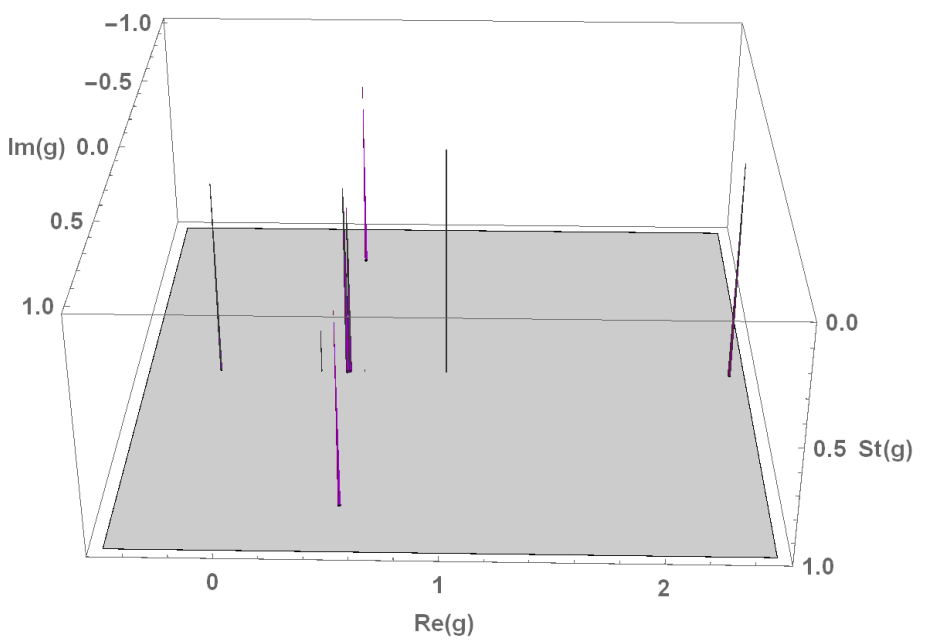

Figure 2: Stability functions of strange fixed points of $M(z, g)$ 


\begin{tabular}{|ll|}
\hline$i$ & $g$ \\
\hline 1 & $-0.139854,2.4276$ \\
2 & -0.139854 \\
3 & $0.515604,0.532281 \pm 0.765252 i$ \\
4 & $0.515604,2.4276$ \\
5 & 0.369621 \\
6 & $0.369621,0.532281 \pm 0.765252 i$ \\
7 & 0.58768 \\
8 & 0.58768 \\
\hline
\end{tabular}

Table 1: $g$-values of $r_{i}(g)$ superattracting fixed points 


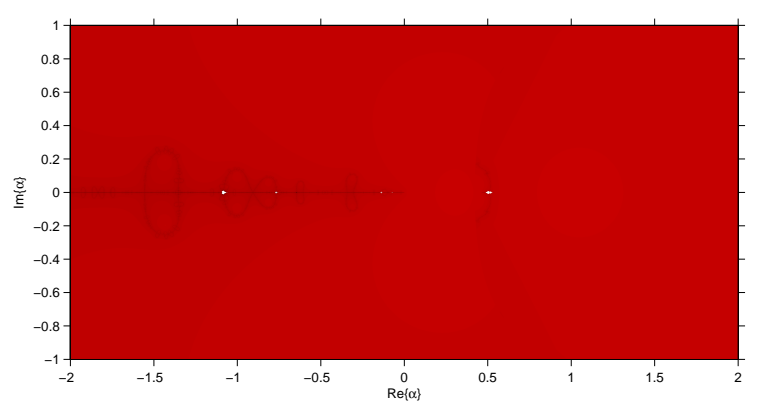

(a) $P_{1}$

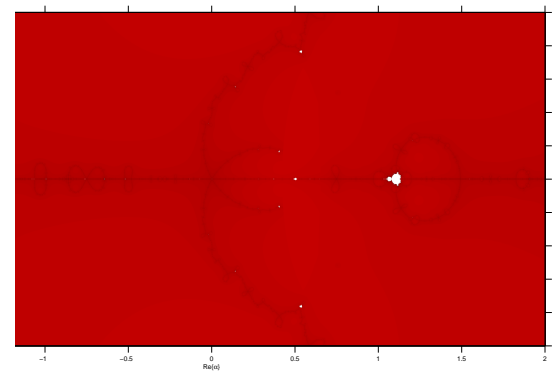

(c) $P_{2}$

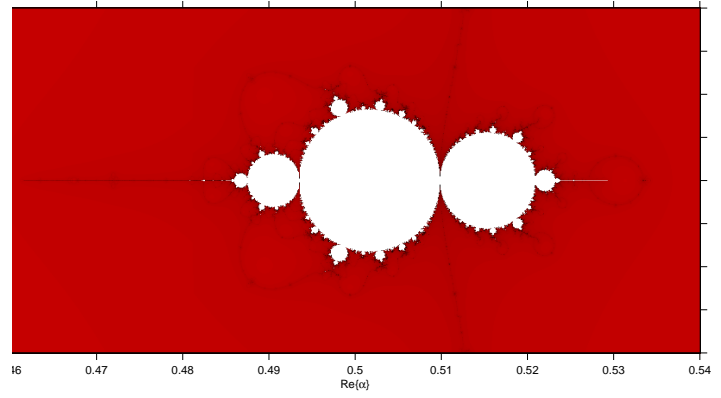

(b) A detail of $P_{1}$

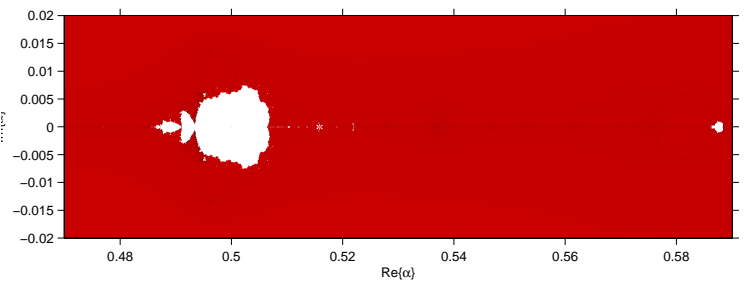

(d) A detail of $P_{2}$

Figure 3: Parameter planes of $M(z, g)$ 


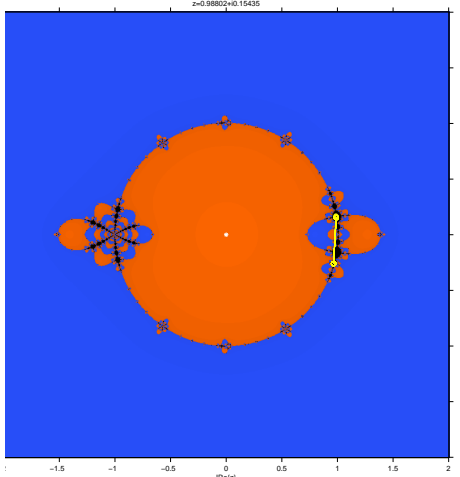

(a) $g=0.4865$

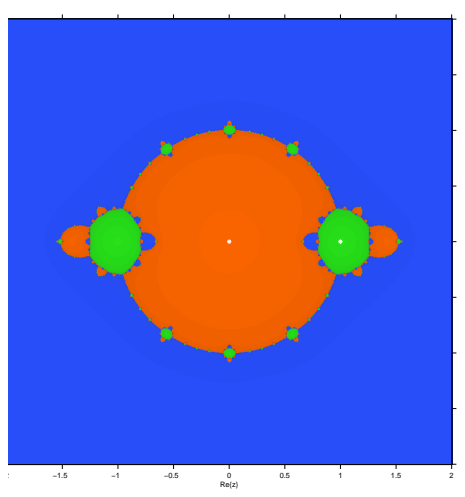

(d) $g=1 / 2$

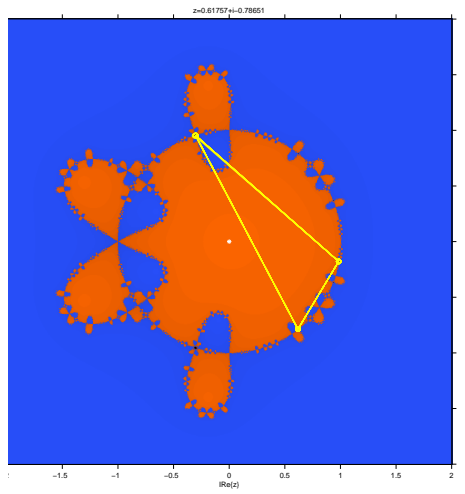

(g) $g=-0.994$

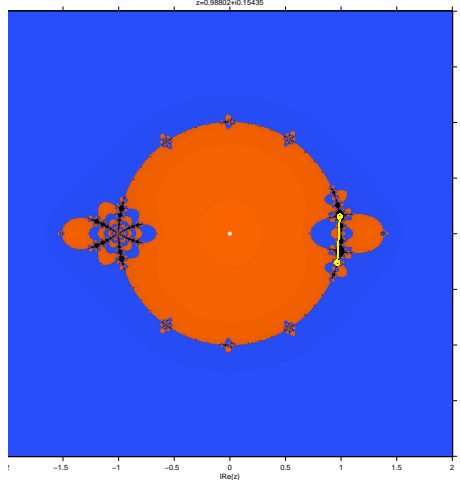

(b) $g=0.4865$

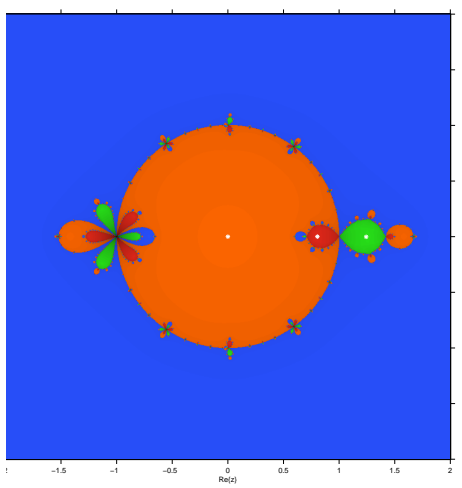

(e) $g=0.515$

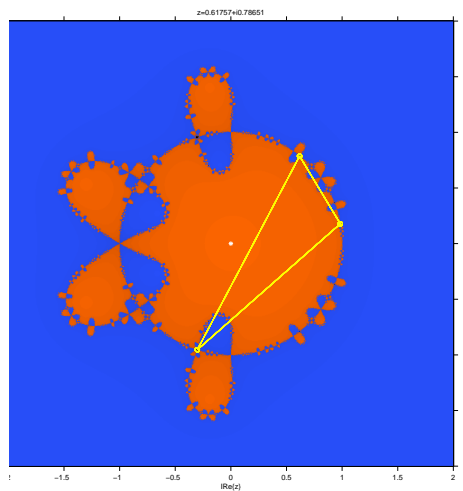

(h) $g=-0.994$

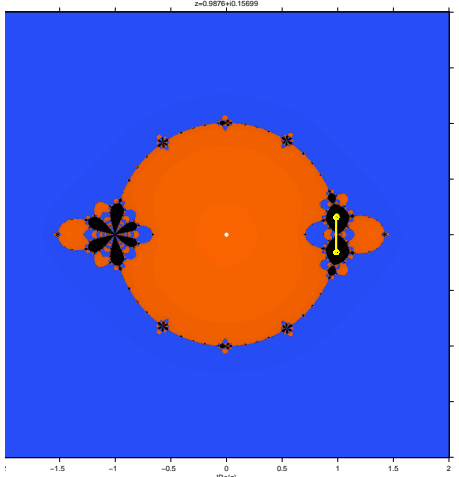

(c) $g=0.49$

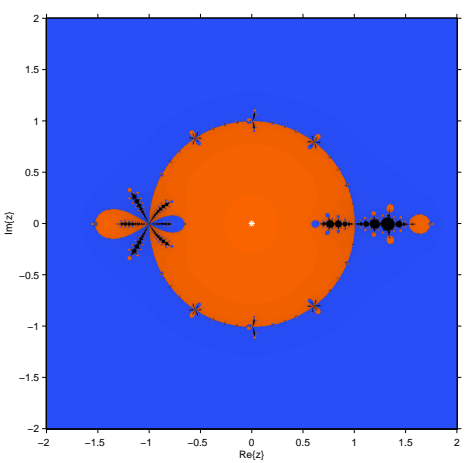

(f) $g=0.5217$

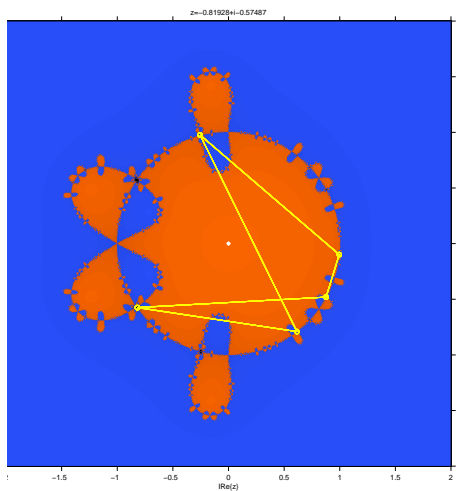

(i) $g=-0.6449+0.0013 i$

Figure 4: Dynamical planes showing unstable behavior 


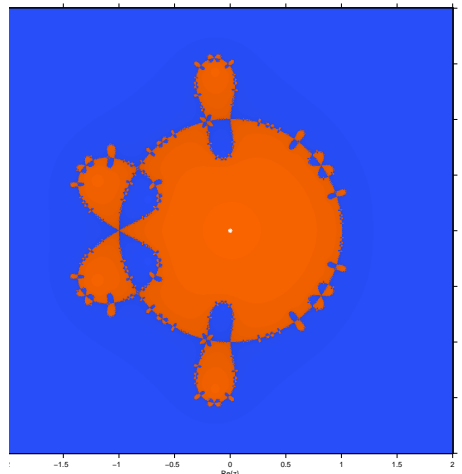

(a) $g=-1 / 2$

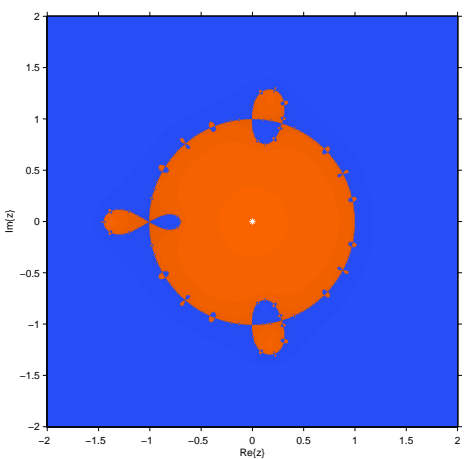

(d) $g=34 / 100$

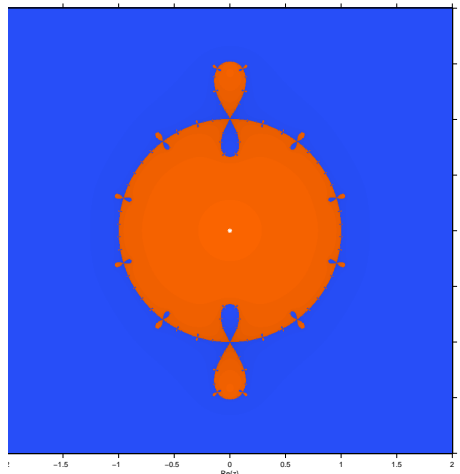

(b) $g=0$

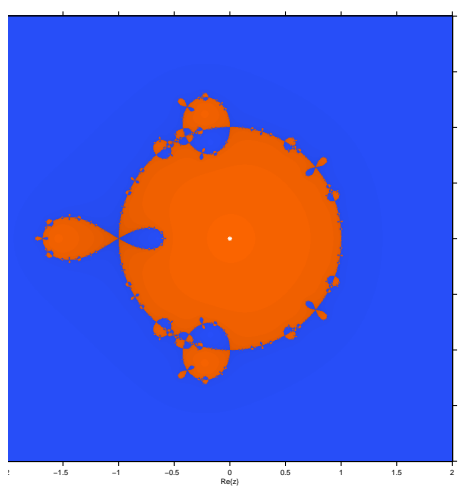

(e) $g=1$

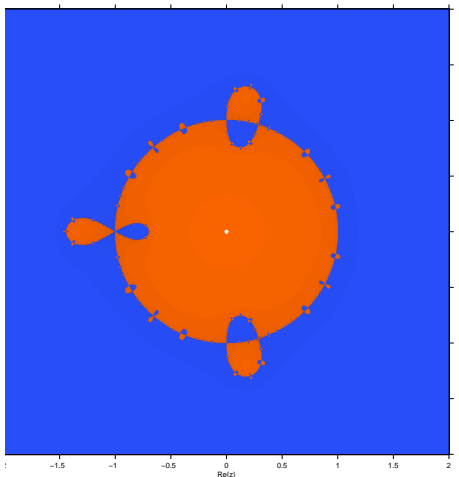

(c) $g=1 / 3$

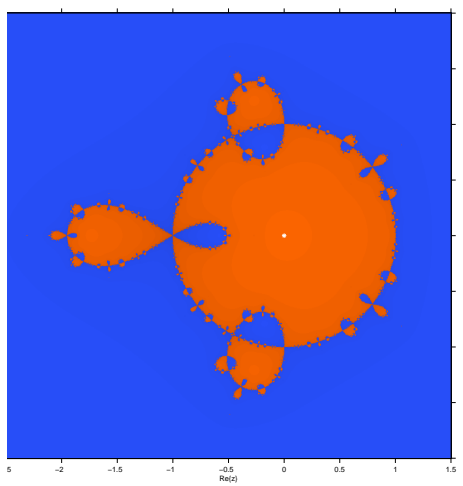

(f) $g=2$

Figure 5: Dynamical planes with stable performance 


\begin{tabular}{|lll|}
\hline$f(x)$ & $Z \operatorname{Zeros}(\xi)$ & Initial guesses \\
\hline$f_{1}(x)=x^{3}-10 ;[32]$ & 2.1544346900318837 & 3.5 \\
$f_{2}(x)=x e^{x^{2}}-\sin ^{2} x+3 \cos x+5 ;[28]$ & -1.2076478271309189 & -1 \\
$f_{3}(x)=e^{-x}+\cos x ;[35]$ & 1.7461395304080124 & 2 \\
$f_{4}(x)=\sin ^{2} x-x^{2}+1 ;[18]$ & 1.4044916482153412 & 2.5 \\
$f_{5}(x)=\tan ^{-1}\left(x^{2}-x\right) ;[10]$ & 0 & -0.6 \\
\hline
\end{tabular}

Table 2: Test problems. 


\begin{tabular}{|c|c|c|c|c|c|c|}
\hline Methods & $n$ & $x_{n}$ & $\left|f\left(x_{n}\right)\right|$ & $\left|x_{n}-x_{n-1}\right|$ & $\frac{x_{n}-x_{n-1}}{\left(x_{n-1}-x_{n-2}\right)^{6}}$ & $\frac{\log \left|\left(x_{n}-x_{n-1}\right) / \eta\right|}{\log \left|x_{n-1}-x_{n-2}\right|}$ \\
\hline \multirow{3}{*}{$S M$} & 1 & 2.14636523057594 & $1.1 e(-1)$ & $8.1 e(-3)$ & & \\
\hline & 2 & 2.15443469003188 & $1.8 e(-14)$ & $1.3 e(-15)$ & 0.001311702775 & 1.7120 \\
\hline & 3 & 2.15443469003188 & $3.6 e(-91)$ & $2.6 e(-92)$ & 0.004805230935 & 6.0000 \\
\hline \multirow{3}{*}{$S H$} & 1 & 2.16376876162794 & $1.3 e(-1)$ & $9.3 e(-3)$ & & \\
\hline & 2 & 2.15443469003189 & $7.3 e(-14)$ & $5.3 e(-15)$ & 0.001639764217 & 5.5262 \\
\hline & 3 & 2.15443469003188 & $2.4 e(-87)$ & $1.7 e(-88)$ & 0.007952539697 & 6.0000 \\
\hline \multirow{3}{*}{$W L M$} & 1 & 2.19798399331210 & $6.2 e(-1)$ & $4.4 e(-2)$ & & \\
\hline & 2 & 2.15443469181772 & $2.5 e(-8)$ & $1.8 e(-9)$ & 0.008938883008 & 6.7964 \\
\hline & 3 & 2.15443469003188 & $1.4 e(-52)$ & $1.0 e(-53)$ & 0.2617905831 & 6.0000 \\
\hline \multirow{3}{*}{$C M$} & 1 & 2.13784204606349 & $2.3 e(-1)$ & $1.7 e(-2)$ & & \\
\hline & 2 & 2.15443469003175 & $1.9 e(-12)$ & $1.3 e(-13)$ & 0.002597471377 & 3.0835 \\
\hline & 3 & 2.15443469003188 & $5.0 e(-79)$ & $3.6 e(-80)$ & 0.006397804388 & 6.0000 \\
\hline \multirow{3}{*}{$M_{6}^{1}$} & 1 & 2.15450202753063 & $9.4 e(-4)$ & $6.7 e(-5)$ & & \\
\hline & 2 & 2.15443469003188 & $6.2 e(-27)$ & $4.5 e(-28)$ & 0.00001134902871 & -14.368 \\
\hline & 3 & 2.15443469003188 & $5.3 e(-166)$ & $3.8 e(-167)$ & 0.004786685025 & 6.0000 \\
\hline \multirow{3}{*}{$M_{6}^{2}$} & 1 & 2.17456584951646 & $2.8 e(-1)$ & $2.0 e(-2)$ & & \\
\hline & 2 & 2.15443469003463 & $3.8 e(-11)$ & $2.8 e(-12)$ & 0.003712956422 & -2.5525 \\
\hline & 3 & 2.15443469003188 & $2.6 e(-70)$ & $1.9 e(-71)$ & 0.04132281370 & 6.0000 \\
\hline \multirow{3}{*}{$M_{6}^{3}$} & 1 & 2.15364064927252 & $1.1 e(-2)$ & $7.9 e(-4)$ & & \\
\hline & 2 & 2.15443469003188 & $2.0 e(-20)$ & $1.4 e(-21)$ & 0.0001333143349 & -6.6100 \\
\hline & 3 & 2.15443469003188 & $6.5 e(-127)$ & $4.7 e(-128)$ & 0.005670253008 & 6.0000 \\
\hline
\end{tabular}

Table 3: Convergence behavior of different methods on test function $f_{1}(x)$. 


\begin{tabular}{|c|c|c|c|c|c|c|}
\hline Methods & $n$ & $x_{n}$ & $\left|f\left(x_{n}\right)\right|$ & $\left|x_{n}-x_{n-1}\right|$ & $\frac{x_{n}-x_{n-1}}{\left(x_{n-1}-x_{n-2}\right)^{6}}$ & $\frac{\log \left|\left(x_{n}-x_{n-1}\right) / \eta\right|}{\log \left|x_{n-1}-x_{n-2}\right|}$ \\
\hline \multirow{3}{*}{$S M$} & 1 & -1.20762432881090 & $4.8 e(-4)$ & $2.3 e(-5)$ & & \\
\hline & 2 & -1.20764782713092 & $4.3 e(-27)$ & $2.1 e(-28)$ & 0.2933373505 & 6.9301 \\
\hline & 3 & -1.20764782713092 & $2.4 e(-165)$ & $1.2 e(-166)$ & 1.265782810 & 6.0000 \\
\hline \multirow{3}{*}{$S H$} & 1 & -1.20756673569784 & $1.6 e(-3)$ & $8.1 e(-5)$ & & \\
\hline & 2 & -1.20764782713092 & $6.1 e(-24)$ & $3.0 e(-25)$ & 1.013977791 & 6.0312 \\
\hline & 3 & -1.20764782713092 & $1.7 e(-146)$ & $8.2 e(-148)$ & 1.064931095 & 6.0000 \\
\hline \multirow{3}{*}{$W L M$} & 1 & -1.07063327000487 & 2.3 & $1.4 e(-1)$ & & \\
\hline & 2 & -1.20937818973602 & $3.5 e(-1)$ & $1.7 e(-3)$ & $1.117277915 e(+6)$ & 2.8172 \\
\hline & 3 & -1.20764782713092 & $4.4 e(-14)$ & $2.2 e(-15)$ & 242.5685996 & 6.000 \\
\hline \multirow{3}{*}{$C M$} & 1 & -1.20762790725150 & $4.0 e(-4)$ & $2.0 e(-5)$ & & \\
\hline & 2 & -1.20764782713092 & $1.6 e(-27)$ & $8.0 e(-29)$ & 0.2486407720 & 7.0405 \\
\hline & 3 & -1.20764782713091 & $6.7 e(-168)$ & $3.3 e(-169)$ & 1.276161529 & 6.0000 \\
\hline \multirow{3}{*}{$M_{6}^{1}$} & 1 & -1.20758594817642 & $1.3 e(-3)$ & $6.2 e(-5)$ & & \\
\hline & 2 & -1.20764782713092 & $1.4 e(-24)$ & $7.1 e(-26)$ & 0.7733128998 & 6.3134 \\
\hline & 3 & -1.20764782713092 & $3.3 e(-150)$ & $1.6 e(-151)$ & 1.265711424 & 6.0000 \\
\hline \multirow{3}{*}{$M_{6}^{2}$} & 1 & -1.20788999265310 & $4.9 e(-3)$ & $2.4 e(-4)$ & & \\
\hline & 2 & -1.20764782713092 & $1.4 e(-24)$ & $7.1 e(-22)$ & 2.999927838 & 6.0963 \\
\hline & 3 & -1.20764782713092 & $8.6 e(-126)$ & $4.2 e(-127)$ & 3.489911931 & 6.0000 \\
\hline \multirow{3}{*}{$M_{6}^{3}$} & 1 & -1.20758224575947 & $1.3 e(-3)$ & $6.6 e(-5)$ & & \\
\hline & 2 & -1.20764782713092 & $2.2 e(-24)$ & $1.1 e(-25)$ & 0.8196704107 & 6.3184 \\
\hline & 3 & -1.20764782713092 & $4.3 e(-149)$ & $2.1 e(-150)$ & 1.352138249 & 6.0000 \\
\hline
\end{tabular}

Table 4: Convergence behavior of different methods on test function $f_{2}(x)$. 


\begin{tabular}{|c|c|c|c|c|c|c|}
\hline Methods & $n$ & $x_{n}$ & $\left|f\left(x_{n}\right)\right|$ & $\left|x_{n}-x_{n-1}\right|$ & $\frac{x_{n}-x_{n-1}}{\left(x_{n-1}-x_{n-2}\right)^{6}}$ & $\frac{\log \left|\left(x_{n}-x_{n-1}\right) / \eta\right|}{\log \left|x_{n-1}-x_{n-2}\right|}$ \\
\hline \multirow{3}{*}{$S M$} & 1 & 1.74613849372402 & $1.2 e(-6)$ & $1.0 e(-6)$ & & \\
\hline & 2 & 1.74613953040801 & $3.5 e(-39)$ & $3.0 e(-39)$ & 0.003873158562 & 5.6629 \\
\hline & 3 & 1.74613953040801 & $2.2 e(-234)$ & $1.9 e(-234)$ & 0.002439747938 & 6.0000 \\
\hline \multirow{3}{*}{$S H$} & 1 & 1.74613809554012 & $1.7 e(-6)$ & $1.4 e(-6)$ & & \\
\hline & 2 & 1.74613953040801 & $3.0 e(-38)$ & $2.4 e(-38)$ & 0.005360764342 & 5.5656 \\
\hline & 3 & 1.74613953040801 & $1.0 e(-228)$ & $8.7 e(-229)$ & 0.002955349157 & 6.0000 \\
\hline \multirow{3}{*}{$W L M$} & 1 & 1.74613259788238 & $8.0 e(-6)$ & $6.9 e(-6)$ & & \\
\hline & 2 & 1.74613953040801 & $8.6 e(-34)$ & $7.4 e(-34)$ & 0.02589702352 & 5.0109 \\
\hline & 3 & 1.74613953040801 & $1.3 e(-201)$ & $1.1 e(-201)$ & 0.006672972055 & 6.0000 \\
\hline \multirow{3}{*}{$C M$} & 1 & 1.74613610285857 & $4.0 e(-6)$ & $3.4 e(-6)$ & & \\
\hline & 2 & 1.74613953040801 & $1.6 e(-35)$ & $1.4 e(-35)$ & 0.01280495544 & 5.6979 \\
\hline & 3 & 1.74613953040801 & $6.5 e(-212)$ & $5.6 e(-212)$ & 0.008462137216 & 6.0000 \\
\hline \multirow{3}{*}{$M_{6}^{1}$} & 1 & 1.74613858497815 & $1.1 e(-6)$ & $9.5 e(-7)$ & & \\
\hline & 2 & 1.74613953040801 & $2.0 e(-39)$ & $1.7 e(-39)$ & 0.003532231323 & 5.7301 \\
\hline & 3 & 1.74613953040801 & $7.9 e(-236)$ & $6.8 e(-239)$ & 0.002439748769 & 6.0000 \\
\hline \multirow{3}{*}{$M_{6}^{2}$} & 1 & 1.74613690212321 & $3.0 e(-6)$ & $2.6 e(-6)$ & & \\
\hline & 2 & 1.74613953040801 & $1.5 e(-36)$ & $1.3 e(-36)$ & 0.009819174353 & 5.3487 \\
\hline & 3 & 1.74613953040801 & $2.5 e(-218)$ & $2.2 e(-218)$ & 0.004020852695 & 6.0000 \\
\hline \multirow{3}{*}{$M_{6}^{3}$} & 1 & 1.74613861287015 & $1.1 e(-6)$ & $9.2 e(-7)$ & & \\
\hline & 2 & 1.74613953040801 & $1.7 e(-9)$ & $1.4 e(-39)$ & 0.003428025949 & 5.7433 \\
\hline & 3 & 1.74613953040801 & $2.5 e(-236)$ & $2.1 e(-236)$ & 0.002411001113 & 6.0000 \\
\hline
\end{tabular}

Table 5: Convergence behavior of different methods on test function $f_{3}(x)$. 


\begin{tabular}{|c|c|c|c|c|c|c|}
\hline Methods & $n$ & $x_{n}$ & $\left|f\left(x_{n}\right)\right|$ & $\left|x_{n}-x_{n-1}\right|$ & $\frac{x_{n}-x_{n-1}}{\left(x_{n-1}-x_{n-2}\right)^{6}}$ & $\frac{\log \left|\left(x_{n}-x_{n-1}\right) / \eta\right|}{\log \left|x_{n-1}-x_{n}\right|}$ \\
\hline \multirow{3}{*}{$S M$} & 1 & 1.41719354720309 & $3.2 e(-2)$ & $1.3 e(-2)$ & & \\
\hline & 2 & 1.40449164821520 & $3.5 e(-13)$ & $1.4 e(-13)$ & 0.007880679099 & -12.708 \\
\hline & 3 & 1.40449164821534 & $6.4 e(-79)$ & $2.6 e(-79)$ & 0.03320320742 & 6.0000 \\
\hline \multirow{3}{*}{$S H$} & 1 & 1.42309768398715 & $4.7 e(-2)$ & $1.9 e(-2)$ & & \\
\hline & 2 & 1.40449164822332 & $2.0 e(-11)$ & $8.0 e(-12)$ & 0.01192877881 & -31.526 \\
\hline & 3 & 1.40449164821534 & $1.3 e(-67)$ & $5.1 e(-68)$ & 0.1923323241 & 6.0000 \\
\hline \multirow{3}{*}{$W L M$} & 1 & 1.43418080615952 & $7.5 e(-2)$ & $3.2 e(-2)$ & & \\
\hline & 2 & 1.40449165076092 & $6.3 e(-9)$ & $2.5 e(-9)$ & 0.02025333849 & -75.771 \\
\hline & 3 & 1.40449164821534 & $3.2 e(-51)$ & $1.3 e(-51)$ & 3.717058573 & 6.0000 \\
\hline \multirow{3}{*}{$C M$} & 1 & 1.42451996292589 & $5.1 e(-2)$ & $2.0 e(-2)$ & & \\
\hline & 2 & 1.40449164821664 & $3.2 e(-12)$ & $1.3 e(-12)$ & 0.01294286097 & -0.075827 \\
\hline & 3 & 1.40449164821534 & $2.2 e(-73)$ & $9.0 e(-74)$ & 0.02013913075 & 6.0000 \\
\hline \multirow{3}{*}{$M_{6}^{1}$} & 1 & 1.41994992005520 & $3.9 e(-2)$ & $1.5 e(-2)$ & & \\
\hline & 2 & 1.40449164821496 & $9.4 e(-13)$ & $3.8 e(-13)$ & 0.009738623585 & -7.5421 \\
\hline & 3 & 1.40449164821534 & $2.5 e(-76)$ & $1.0 e(-76)$ & 0.02763118957 & 6.0000 \\
\hline \multirow{3}{*}{$M_{6}^{2}$} & 1 & 1.42684791179265 & $5.6 e(-2)$ & $2.2 e(-2)$ & & \\
\hline & 2 & 1.40449164830403 & $2.2 e(-10)$ & $8.9 e(-11)$ & 0.01463630900 & -48.989 \\
\hline & 3 & 1.40449164821534 & $9.4 e(-61)$ & $3.8 e(-61)$ & 0.7103333905 & 6.0000 \\
\hline \multirow{3}{*}{$M_{6}^{3}$} & 1 & 1.41974860211107 & $3.8 e(-2)$ & $1.5 e(-2)$ & & \\
\hline & 2 & 1.40449164821481 & $1.3 e(-12)$ & $5.3 e(-13)$ & 0.009601051782 & -13.060 \\
\hline & 3 & 1.40449164821534 & $2.7 e(-75)$ & $1.1 e(-75)$ & 0.04181253514 & 6.0000 \\
\hline
\end{tabular}

Table 6: Convergence behavior of different methods on test function $f_{4}(x)$. 


\begin{tabular}{|c|c|c|c|c|c|c|}
\hline Methods & $n$ & $x_{n}$ & $\left|f\left(x_{n}\right)\right|$ & $\left|x_{n}-x_{n-1}\right|$ & $\frac{x_{n}-x_{n-1}}{\left(x_{n-1}-x_{n-2}\right)^{6}}$ & $\frac{\log \left|\left(x_{n}-x_{n-1}\right) / \eta\right|}{\log \left|x_{n-1}-x_{n-2}\right|}$ \\
\hline \multirow{3}{*}{$S M$} & 1 & 0.00577787122343893 & $5.7 e(-3)$ & $5.8 e(-3)$ & & \\
\hline & 2 & $-1.49034025294135 e(-14)$ & $1.5 e(-14)$ & $1.5 e(-14)$ & 0.1169196293 & 8.4567 \\
\hline & 3 & $-4.46417807748464 e(-84)$ & $4.5 e(-84)$ & $4.5 e(-84)$ & 0.4005698732 & 6.0000 \\
\hline \multirow{3}{*}{$S H$} & 1 & $5.62325793192393 e(-3)$ & $5.6 e(-3)$ & $5.6 e(-3)$ & & \\
\hline & 2 & $-5.35466783046316 e(-14)$ & $5.4 e(-14)$ & $5.4 e(-14)$ & 0.1139653251 & 11.381 \\
\hline & 3 & $-3.92865561590856 e(-80)$ & $3.9 e(-80)$ & $3.9 e(-80)$ & 1.693575288 & 6.0000 \\
\hline \multirow{3}{*}{$W L M$} & 1 & 0.00622955998102951 & $6.2 e(-3)$ & $6.2 e(-3)$ & & \\
\hline & 2 & $-1.23264756657509 e(-12)$ & $1.2 e(-12)$ & $1.2 e(-12)$ & 0.1254973971 & 16.238 \\
\hline & 3 & $-6.82070199938708 e(-71)$ & $6.8 e(-71)$ & $6.8 e(-71)$ & 21.09087956 & 6.0000 \\
\hline \multirow{3}{*}{$C M$} & 1 & 0.00677840358329132 & $6.7 e(-3)$ & $6.8 e(-3)$ & & \\
\hline & 2 & $-7.86679175179491 e(-14)$ & $7.9 e(-14)$ & $7.9 e(-14)$ & 0.1358146857 & 9.5769 \\
\hline & 3 & $-1.93127820428429 e(-79)$ & $1.9 e(-79)$ & $1.9 e(-79)$ & 0.8110228976 & 6.0000 \\
\hline \multirow{3}{*}{$M_{6}^{1}$} & 1 & $5.53918581656025 e(-3)$ & $5.5 e(-3)$ & $5.5 e(-3)$ & & \\
\hline & 2 & $-1.11369585269096 e(-14)$ & $1.1 e(-14)$ & $1.1 e(-14)$ & 0.1123550038 & 8.4580 \\
\hline & 3 & $-7.77371358320307 e(-85)$ & $7.8 e(-85)$ & $7.8 e(-85)$ & 0.3855591352 & 6.0106 \\
\hline \multirow{3}{*}{$M_{6}^{2}$} & 1 & $5.83233867129775 e(-3)$ & $5.8 e(-3)$ & $5.8 e(-3)$ & & \\
\hline & 2 & $-1.84117995134919 e(-13)$ & $1.8 e(-13)$ & $1.8 e(-13)$ & 0.1179581693 & 13.344 \\
\hline & 3 & $-1.74581825115534 e(-76)$ & $1.7 e(-76)$ & $1.7 e(-76)$ & 4.677780398 & 6.0000 \\
\hline \multirow{3}{*}{$M_{6}^{3}$} & 1 & $5.53444465971672 e(-3)$ & $5.5 e(-3)$ & $5.5 e(-3)$ & & \\
\hline & 2 & $-8.86422521017536 e(-15)$ & $8.9 e(-15)$ & $8.9 e(-15)$ & 0.1122641096 & 8.0148 \\
\hline & 3 & $-1.61704994597297 e(-85)$ & $1.6 e(-85)$ & $1.6 e(-85)$ & 0.3084583182 & 6.0000 \\
\hline
\end{tabular}

Table 7: Convergence behavior of different methods on test function $f_{5}(x)$. 


\begin{tabular}{|c|c|c|c|c|c|}
\hline Methods & $k$ & $\left\|F\left(x^{(k)}\right)\right\|$ & $\left\|x^{(k)}-x^{(k-1)}\right\|$ & $\frac{\left\|x^{(k)}-x^{(k-1)}\right\|}{\left\|x^{(k-1)}-x^{(k-2)}\right\|^{6}}$ & $\frac{\log \left\|\left(x^{(k)}-x^{(k-1)}\right) / \eta\right\|}{\log \left\|x^{(k-1)}-x^{(k-2)}\right\|}$ \\
\hline \multirow{3}{*}{$H M_{6}$} & 1 & 7.9 & 3.2 & & \\
\hline & 2 & $2.0 e(-1)$ & $8.0 e(-2)$ & $4.655989498 e(-7)$ & 4.0943 \\
\hline & 3 & $6.4 e(-10)$ & $1.3 e(-10)$ & 0.00006937678770 & 6.0000 \\
\hline \multirow{3}{*}{$C M_{6}^{1}$} & 1 & $6.5 e(-1)$ & $1.3 e(-1)$ & & \\
\hline & 2 & $1.3 e(-8)$ & $2.6 e(-9)$ & 0.001824327331 & 7.6448 \\
\hline & 3 & $3.6 e(-55)$ & $8.3 e(-56)$ & 0.0005675860446 & 6.0000 \\
\hline \multirow{3}{*}{$C M_{6}^{2}$} & 1 & $5.3 e(+5)$ & $1.2 e(+2)$ & & \\
\hline & 2 & $3.7 e(+4)$ & 50 & $1.307493606 e(-12)$ & 5.5409 \\
\hline & 3 & $2.6 e(+3)$ & 20 & $1.532153278 e(-11)$ & 6.0000 \\
\hline \multirow{3}{*}{$S M_{6}$} & 1 & $2.9 e(+9)$ & $2.2 e(+3)$ & & \\
\hline & 2 & $2.5 e(+8)$ & $9.5 e(+2)$ & $6.784488805 e(-19)$ & 5.6802 \\
\hline & 3 & $2.1 e(+7)$ & $4.2 e(+2)$ & $9.492439643 e(-18)$ & 6.0000 \\
\hline \multirow{3}{*}{$\bar{M}_{6}^{1}$} & 1 & $5.4 e(-1)$ & $1.3 e(-1)$ & & \\
\hline & 2 & $2.5 e(-9)$ & $8.8 e(-10)$ & 0.003838994783 & 11.475 \\
\hline & 3 & $4.6 e(-59)$ & $2.5 e(-59)$ & 0.0001496116349 & 6.0000 \\
\hline \multirow{3}{*}{$\bar{M}_{6}^{2}$} & 1 & 2.4 & $4.6 e(-1)$ & & \\
\hline & 2 & $3.8 e(-5)$ & $7.2 e(-6)$ & 0.002871441515 & 7.6366 \\
\hline & 3 & $4.2 e(-34)$ & $9.0 e(-35)$ & 0.0007170104768 & 6.0000 \\
\hline \multirow{3}{*}{$\bar{M}_{6}^{3}$} & 1 & $5.7 e(-1)$ & $1.4 e(-1)$ & & \\
\hline & 2 & $4.3 e(-9)$ & $1.3 e(-9)$ & 0.004060081644 & 11.379 \\
\hline & 3 & $1.0 e(-57)$ & $4.0 e(-58)$ & 0.0001698889314 & 6.0000 \\
\hline
\end{tabular}

Table 8: Convergence behavior of different methods on Van der Pol equation.

\begin{tabular}{|ccc|}
\hline$i$ & $\psi_{i}$ & $\phi_{i}$ \\
\hline 0 & 1.3954170041747090114 & 1.7461756494150842271 \\
1 & 1.7444828545735749268 & 2.0364691127919609051 \\
2 & 2.0656234369405315689 & 2.2390977868265978920 \\
3 & 2.4600678478912500533 & 2.4600678409809344550 \\
\hline
\end{tabular}

Table 9: The values of $\psi_{i}$ and $\phi_{i}$ (in radians) for Example 2 . 


\begin{tabular}{|c|c|c|c|c|c|}
\hline Methods & $k$ & $\left\|F\left(x^{(k)}\right)\right\|$ & $\left\|x^{(k)}-x^{(k-1)}\right\|$ & $\frac{\left\|x^{(k)}-x^{(k-1)}\right\|}{\left\|x^{(k)}-x^{(k-1)}\right\|^{6}}$ & $\frac{\log \left\|\left(x^{(k)}-x^{(k-1)}\right) / \eta\right\| \|}{\log \left\|x^{(k-1)}-x^{(k-2)}\right\|}$ \\
\hline \multirow{3}{*}{$H M_{6}$} & 1 & $3.0 e(-4)$ & $7.0 e(-3)$ & & \\
\hline & 2 & $8.7 e(-9)$ & $9.3 e(-7)$ & 76.33255933 & 13.446 \\
\hline & 3 & $1.4 e(-28)$ & $5.2 e(-27)$ & $7.812154542 e(+6)$ & 6.0000 \\
\hline \multirow{3}{*}{$C M_{6}^{1}$} & 1 & $9.9 e(-5)$ & $4.9 e(-3)$ & & \\
\hline & 2 & $8.2 e(-11)$ & $5.2 e(-9)$ & 52.84832345 & 11.730 \\
\hline & 3 & $1.2 e(-47)$ & $4.9 e(-46)$ & 375218.7071 & 6.0000 \\
\hline \multirow{3}{*}{$C M_{6}^{2}$} & 1 & $1.9 e(-4)$ & $2.5 e(-3)$ & & \\
\hline & 2 & $5.4 e(-11)$ & $4.3 e(-9)$ & 27.24757306 & 14.678 \\
\hline & 3 & $2.7 e(-47)$ & $7.3 e(-46)$ & $1.930582801 e(+7)$ & 6.0000 \\
\hline \multirow{3}{*}{$S M_{6}$} & 1 & $7.5 e(-4)$ & $4.9 e(-3)$ & & \\
\hline & 2 & $3.3 e(-9)$ & $6.5 e(-7)$ & 58.14341084 & 14.714 \\
\hline & 3 & $1.7 e(-33)$ & $7.7 e(-32)$ & $4.841793597 e(+7)$ & 6.0000 \\
\hline \multirow{3}{*}{$\bar{M}_{6}^{1}$} & 1 & $6.9 e(-5)$ & $5.2 e(-3)$ & & \\
\hline & 2 & $1.9 e(-12)$ & $2.8 e(-8)$ & 55.45963282 & 9.5654 \\
\hline & 3 & $1.4 e(-56)$ & $2.0 e(-54)$ & 13671.16552 & 6.0000 \\
\hline \multirow{3}{*}{$\bar{M}_{6}^{2}$} & 1 & $1.9 e(-4)$ & $4.6 e(-3)$ & & \\
\hline & 2 & $1.5 e(-10)$ & $1.2 e(-10)$ & 50.59058423 & 12.507 \\
\hline & 3 & $4.1 e(-45)$ & $1.6 e(-43)$ & $1.214170932 e(+6)$ & 6.0000 \\
\hline \multirow{3}{*}{$\bar{M}_{6}^{3}$} & 1 & $7.3 e(-5)$ & $5.2 e(-3)$ & & \\
\hline & 2 & $1.5 e(-12)$ & $2.2 e(-10)$ & 55.49203506 & 9.4080 \\
\hline & 3 & $4.1 e(-57)$ & $5.1 e(-55)$ & 10723.07345 & 6.0000 \\
\hline
\end{tabular}

Table 10: Convergence behavior of different methods kinematic synthesis problem. 


\begin{tabular}{|c|c|c|c|c|c|}
\hline Methods & $k$ & $\left\|F\left(x^{(k)}\right)\right\|$ & $\left\|x^{(k)}-x^{(k-1)}\right\|$ & $\frac{\left\|x^{(k)}-x^{(k-1)}\right\|}{\left\|x^{(k)}-x^{(k-1)}\right\|^{6}}$ & $\frac{\log \left\|\left(x^{(k)}-x^{(k-1)}\right) / \eta\right\|}{\log \left\|x^{(k-1)}-x^{(k-2)}\right\|}$ \\
\hline \multirow{3}{*}{$H M_{6}$} & 1 & $9.5 e(-3)$ & 1.1 & & \\
\hline & 2 & $6.3 e(-15)$ & $3.2 e(-3)$ & 0.001649635600 & -59.727 \\
\hline & 3 & $8.5 e(-76)$ & $2.1 e(-15)$ & 2.095048920 & 6.0000 \\
\hline \multirow{3}{*}{$C M_{6}^{1}$} & 1 & $1.5 e(-3)$ & $4.9 e(-4)$ & & \\
\hline & 2 & $2.6 e(-23)$ & $8.7 e(-24)$ & 0.0002522868775 & -2.1037 \\
\hline & 3 & $8.3 e(-142)$ & $2.8 e(-142)$ & 0.0006208888614 & 6.0000 \\
\hline \multirow{3}{*}{$C M_{6}^{2}$} & 1 & $6.6 e(-3)$ & $2.2 e(-3)$ & & \\
\hline & 2 & $1.9 e(-18)$ & $6.4 e(-19)$ & 0.001141948774 & -8.4606 \\
\hline & 3 & $1.1 e(-111)$ & $3.8 e(-112)$ & 0.005571101903 & 6.0000 \\
\hline \multirow{3}{*}{$S M_{6}$} & 1 & $1.2 e(-2)$ & $4.1 e(-3)$ & & \\
\hline & 2 & $2.0 e(-16)$ & $6.7 e(-17)$ & 0.002133062120 & -11.861 \\
\hline & 3 & $4.1 e(-99)$ & $1.4 e(-99)$ & 0.01466056139 & 6.0000 \\
\hline \multirow{3}{*}{$\bar{M}_{6}^{1}$} & 1 & $1.1 e(-4)$ & $4.8 e(-5)$ & & \\
\hline & 2 & $4.4 e(-30)$ & $1.5 e(-30)$ & 0.00002443680628 & -8.5687 \\
\hline & 3 & $3.8 e(-183)$ & $1.3 e(-183)$ & 0.0001242343978 & 6.0000 \\
\hline \multirow{3}{*}{$\bar{M}_{6}^{2}$} & 1 & $2.2 e(-3)$ & $7.3 e(-4)$ & & \\
\hline & 2 & $5.3 e(-22)$ & $1.8 e(-22)$ & 0.0003777843031 & -3.7757 \\
\hline & 3 & $1.0 e(-133)$ & $3.3 e(-134)$ & 0.001117210881 & 6.0000 \\
\hline \multirow{3}{*}{$\bar{M}_{6}^{3}$} & 1 & $2.1 e(-4)$ & $6.9 e(-5)$ & & \\
\hline & 2 & $4.7 e(-29)$ & $1.6 e(-29)$ & 0.00003527975367 & -6.7734 \\
\hline & 3 & $6.7 e(-177)$ & $2.2 e(-177)$ & 0.0001468257053 & 6.0000 \\
\hline
\end{tabular}

Table 11: Convergence behavior of different methods on Example 3. 